\title{
Bosonization on higher genus Riemann surfaces
}

\section{Citation}

Alvarez-Gaumé, Luis, Jean-Benoît Bost, Gregory Moore, Philip Nelson, and Cumrun Vafa. 1987. "Bosonization on Higher Genus Riemann Surfaces." Communications in Mathematical Physics 112 (3): 503-52. https://doi.org/10.1007/bf01218489.

\section{Permanent link}

http://nrs.harvard.edu/urn-3:HUL.InstRepos:41385070

\section{Terms of Use}

This article was downloaded from Harvard University's DASH repository, and is made available under the terms and conditions applicable to Other Posted Material, as set forth at http:// nrs.harvard.edu/urn-3:HUL.InstRepos:dash.current.terms-of-use\#LAA

\section{Share Your Story}

The Harvard community has made this article openly available.

Please share how this access benefits you. Submit a story.

\section{Accessibility}




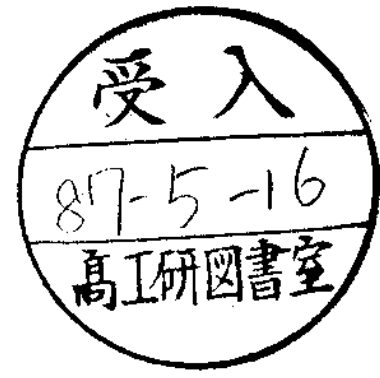

BOSONIZATION ON HIGHER GENUS RIEMANN SURFACES ${ }^{*}$ )

L. Alvarez-Gaumé" )+)

CERN -- Geneva

J. -B. Bost

Ecole Normale Supérieure - Paris

G. Moore ${ }^{\circ}$ ), Ph. Nelson $\left.{ }^{\circ}\right)+$ ) and $\mathrm{C} \cdot \operatorname{Vafa}^{\circ}$ )

Lyman Laboratory of Physics, Harvard University Cambridge

\section{A B S S T R A}

We prove the equivalence between certain fermionic and bosonic theories in two space-time dimensions. The theories have fields of arbitrary spin on compact surfaces with any number of handles. Global considerations require that we add new topological terms to the bosonic action. The proof that our prescription is correct relies on methods of complex algebraic geometry.

*) Work supported in part by NSF grant PHY-8215249 and DOE contract DE-FG02-84-ER-40164$\mathrm{A001}$.

") Alfred P. Sloan Foundation Fellow.

+ Address after 1 July 1987: Department of Physics, Boston University, Boston MA 02215 U.S.A.

-) Harvard Society of Fellows. 


\section{Introduction}

Two-dimensional quantum field theory is very special. Many surprising and beautiful results turn out to be true only in two dimensions, including for example the exact solvability of certain models, the equivalence of fermionic and bosonic field theories, and so on. One way of describing the root cause for all these miracles is to note that in two dimensions the light cone is disconnected: it consists of a left moving and a right moving branch, and massless particles stay on one branch or the other. 1

This cleavage in turn comes from the fact that in two dimensions the scalar wave operator factorizes into the product of left and right moving derivatives. In euclidean space the analogous statement is

$$
\nabla^{2}=\bar{\partial}^{\dagger} \bar{\partial}
$$

where $\bar{\partial}$ is the Cauchy-Riemann operator. Thus in a sense we can say that $2 d$ fields are special because for them complex analysis plays a key role.

In this paper we will see how complex analytic methods can extend our understanding of $2 d$ fields from surfaces with the topology of the plane (or sphere) to arbitrary compact euclidean spacetimes. Specifically we will study Fermi-Bose equivalence, or "bosonization." We give a prescription for bosonizing the correlation functions of a first order fermionic system with fields of any spin and any twisted spin structure. Our prescription generalizes that of [2][3][4]; in particular certain global terms must be added to the scalar action for nontrivial spacetimes. Most of these results were announced in [5] and build on [6] and [7].

Field theory on complicated surfaces, and in particular bosonization, has become an important tool in the study of string theory. For example, bosonization has been used in light-cone gauge to prove the equivalence of the Green-Schwarz and NSR superstring [8][9]. Bosonization also plays a key role in understanding the gauge- and super-symmetry of the heterotic string [10] and in formulating the covariant fermion emission vertex [11][12]. The methods we use however are quite general and we expect them to be of use in $2 d$ field theory for problems other than bosonization. For instance we obtain some expressions for functional determinants in terms of the natural functions associated to a Riemann surface.

The key step in understanding $2 d$ fields on compact surfaces is the observation that while the amplitudes are functionals on the large space of metric background fields, nev-

\footnotetext{
1 See, e.g. the physical discussion in $\S V . B$ of $[1]$.
} 
ertheless most of this dependence is understood using the various known anomalies. The only interesting dependence is on the "moduli space" $M_{g}$ of conformally-inequivalent surfaces with $g$ handles. Similarly the dependence on flat background gauge fields boils down to one on the "jacobian variety" $J(\Sigma)$ of inequivalent bundles on a given surface $\Sigma$. The spaces $\mathcal{M}_{g}$ and $J(\Sigma)$ are both finite-dimensional. (Indeed both are trivial on the plane, corresponding to the well-known fact that fermion dynamics on the plane is completely given by the anomaly.) Furthermore each is naturally a complex space, a consequence of the complex form of the wave operator (1.1). Thus as mentioned earlier, powerful complexanalytic methods are available to study quantum amplitudes. This is why two dimensions is so special.

The link between field-theoretic and algebraic-geometric methods is provided by the theorems in [13][14] [15] (see also [16] [17] [18]), which describe the determinant of a family of Cauchy-Riemann operators in terms of the complex structure of $\mathcal{M}_{g}$. The case originally studied by Quillen involves families of operators at one point of $\mathcal{M}_{g}$, parametrized by background gauge potentials on the given surface. These results were later generalized by Belavin and Knizhnik and by Bost and Jolicœur (using results of Bismut and Freed) to include families of Cauchy-Riemann operators parametrized by $\mathcal{M}_{g}$, which are of interest in string theory. The main conclusion is that the combinations of determinants appearing in the integrand over $\mathcal{M}_{g}$ in the bosonic and fermionic strings factorize into sections of flat holomorphic line bundles on $\mathcal{M}_{g}$. This factorization is useful for example when we study the infinities of string theory by allowing Riemann surfaces with nodes.

It has also been known for some time that Quillen's work is closely related to Falting's work on Arakelov geometry [19][20]. It was suggested in [15] that a combination of Quillen's and Faltings' ideas would be of use in string theory. We will use just such a combination to prove our results on bosonization.

As mentioned, we will generalize the bosonization prescription given in $[2][3][4]$ for anticommuting fields of any spin on the sphere. When we try to generalize to arbitrary compact surfaces, however, we face the problem that there is in general no euclidean "time" to use in a canonical formalism. Fortunately there is one case, the torus, where operator methods still work and yet the topology is interesting enough to show what happens in higher loops. We will use the canonical formalism to get the correct prescription in a 
simple case, then use modular invariance and factorization to guess the correct general prescription, in any number of loops. The prescription so obtained is unique.

To prove that our bosonization rules really do work, we will compute corresponding quantities in the bosonic and fermionic languages. Setting these equal gives a set of identities which express the content of Fermi-Bose equivalence. Finally we prove the identities using methods of algebraic geometry, thus establishing bosonization.

While the last steps get rather involved, we emphasize that the prescription itself is not too complicated. The reader may wish to turn immediately to Section 4 to see the statement of the bosonization rules.

Throughout this paper we will discuss only nonchiral theories. We restrict to this case because, as is well known, chiral determinants are problematic in $2 d$ gravity due to anomalies. In the bosonic language this appears as a difficulty in defining chiral scalar fields in a path integral. There has been some progress in chiral bosonization in [21] [22] [23][24] and elsewhere, but a discussion is beyond the scope of this paper.

Also, in this paper we discuss bosonization physically in terms of path integrals and mathematically in terms of isometries of determinant bundles. Historically, another approach to bosonization in spin $1 / 2$ has proceeded via the isomorphism between spinor and vertex operator representations of affine Lie algebras [25][26]. It would be extremely interesting to unify and generalize these two approaches using a general operator formalism. Recent progress on this problem has been made along these lines in [27] [24][28].

In sect. 2 we describe various aspects of the theory of Riemann surfaces which we will need. In particular we discuss various ways to describe bundles, choices of homology basis, holonomy, and Arakelov metrics. References [6][16][29][17] may be useful background for this section and for the whole paper. In Section 3 we arrive at the bosonization rules and in particular show that the scalar action is independent of various choices made in defining it. In Section 4 we give the complete set of rules, and work out the identities mentioned above. Section 5 contains the proofs of these identities, and we conclude in Section 6.

We draw the reader's attention to several preprints on related topics which we received after this work was completed. These include $[30][22][23][31]-[34]$. 


\section{Foundations}

This is a long introductory section in which we introduce some machinery. ${ }^{2}$ In particular we describe bundles over Riemann surfaces in three different ways: via transition functions, via divisors, and via points in the jacobian mentioned earlier. We also briefly review theta functions and Arakelov metrics. The reader may wish to skip this section and refer to it as needed.

\section{A. Surfaces}

Let $\Sigma$ be a smooth compact connected surface. We will always assume $\Sigma$ is oriented, as for example in the heterotic string. We will soon need to give $\Sigma$ more structure, but first we will briefly note some facts about its topology.

The homology of $\Sigma$ is simple. $H_{0}(\Sigma ; Z)$ has one generator since $\Sigma$ is connected, while $H_{2}(\Sigma ; Z)$ has one generator since $\Sigma$ is compact, connected, and oriented [35]. The first homology group has $2 g$ generators, where the genus $g$ is an integer which completely specifies $\Sigma$ topologically. See Fig. 1. By triangulating $\Sigma$ one can see that the Euler number of $\Sigma$ is $\chi=2-2 g ; \chi$ is also the Chern number of the tangent to $\Sigma$. The oriented intersection number of two 1-cycles is a signed integer, and

$$
a \cdot a^{\prime}=-a^{\prime} \cdot a
$$

This pairing defines a quadratic form on $H_{1}(\Sigma)$.

We will want our amplitudes to depend only on intrinsic information. For example, the partition function for spin-1/2 fermions depends on a surface and a choice of spin bundle. In practice, however, we need coordinates to describe the intrinsic data, and this requires that we make some noninvariant choices. We then have to verify later that our answers are independent of the choices made. The most important such choice, which we will use throughout this paper, is that of a basis of $H_{1}(\Sigma ; Z)$. While there is no preferred basis, we can restrict the choice somewhat by choosing only bases of the form $A=\left\{a_{1}, \ldots, a_{g}, b_{1}, \ldots b_{g}\right\}$, satisfying the invariant condition:

$$
a_{i} \cdot a_{j}=b_{i} \cdot b_{j}=0, \quad a_{i} \cdot b_{j}=\delta_{i j},
$$

2 We thank V. Dellapietra for many discussions on the material in this section, and also section 3.C. 


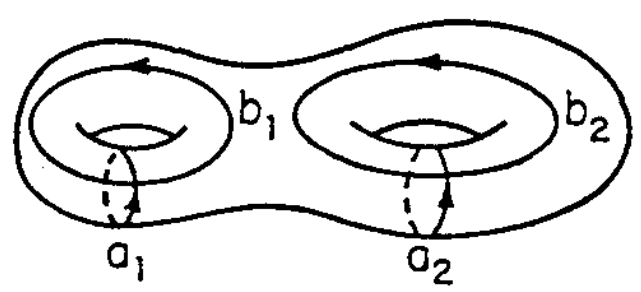

Fig. 1: A Riemann surface of genus two, with a canonical homology basis.

or

$$
\left(\begin{array}{l}
\vec{a} \\
\vec{b}
\end{array}\right) \cdot(\vec{a}, \vec{b})=J \quad \text { where } \quad J \equiv\left(\begin{array}{cc}
0 & 1 \\
-1 & 0
\end{array}\right)
$$

Any basis $A$ with property (2.1) will be called "canonical". Any other canonical basis $\tilde{A}$ will then be related to $A$ by an integer matrix preserving $J$ :

$$
\tilde{A}=A \cdot \Lambda^{-1}, \quad \Lambda^{t} J \Lambda=J .
$$

The group of such $\Delta$ is the "symplectic modular group" $S p(2 g, \mathrm{Z})$. Letting

$$
\Delta=\left(\begin{array}{cc}
A & -B \\
-C & D
\end{array}\right)
$$

we get

$$
\Lambda^{-1}=\left(\begin{array}{ll}
D^{t} & B^{t} \\
C^{t} & A^{t}
\end{array}\right)
$$

We can also define a dual basis $A^{*}$ of $H^{1}(\Sigma ; Z)$ by

$$
\begin{gathered}
A^{*}=\left\{\alpha^{1}, \ldots, \alpha^{g}, \beta^{1}, \ldots, \beta^{g}\right\}^{t} \\
\left\langle A^{*}, A\right\rangle=1 \\
\tilde{A}^{*}=\Lambda A^{*} .
\end{gathered}
$$



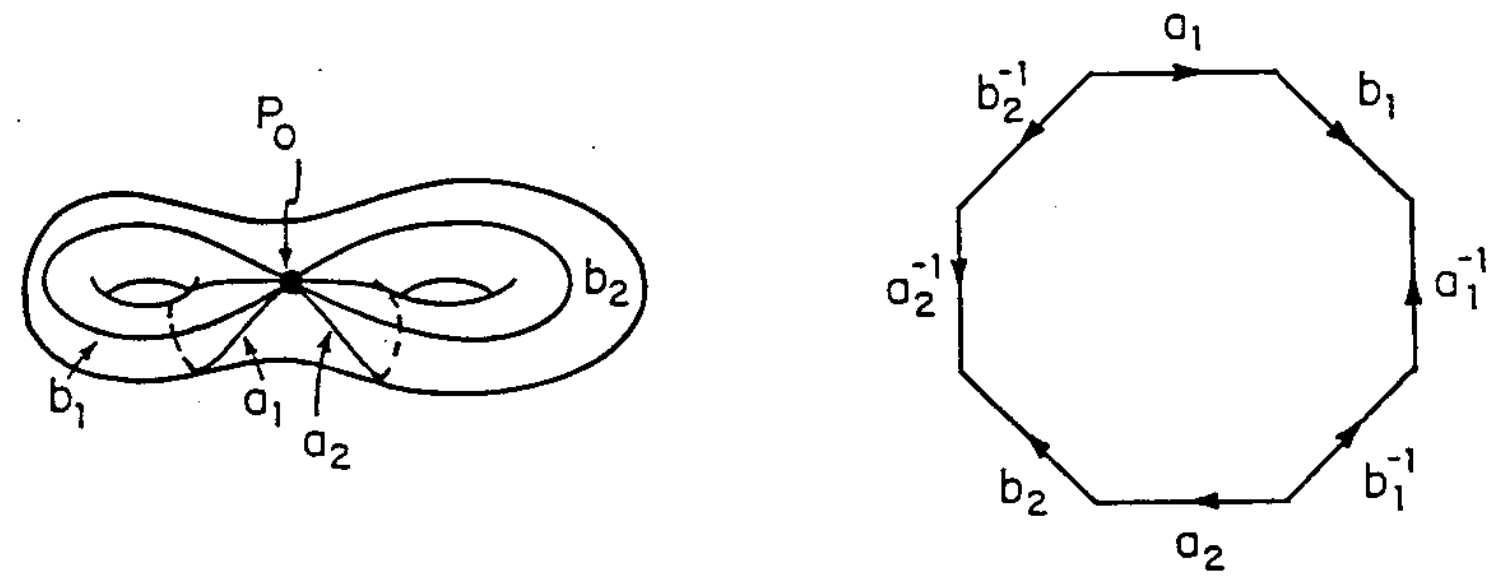

Fig. 2: a) Representatives of a basis intersecting at $P_{0}$. b) The cut surface $\Sigma_{c}$.

Thus if we expand a cohomology class $\psi$ as $\psi=(n, m)\left(\begin{array}{l}\alpha \\ \beta\end{array}\right)$, then with respect to a new basis $\psi$ is described by

$$
(\widetilde{n}, \tilde{m})=(n, m) \Lambda^{-1}
$$

We will usually append a subscript $A$ to any object we construct which depends on a choice of basis.

Again, the introduction of a basis $A$ is a necessary evil needed to parametrize various spaces. It explicitly breaks invariance under diffeomorphisms, since $f_{*}\left(a_{i}\right)$ is not in general homologous to $a_{i}$ if $f$ is not connected to the identity. However, since $f_{*}(a) \cdot f_{*}\left(a^{\prime}\right)=a \cdot a^{\prime}$, we do know that $f_{*} A$ always differs from $A$ by a transformation in $S p(2 g, \mathrm{Z})$. Thus if we are careful not to make any further noninvariant choices beyond that of $A$, we see that invariance under $S p(2 g, Z)$ suffices to establish invariance under the full group of disconnected coordinate transformations of $\Sigma$. The former condition is also called "modular invariance."

Given a homology basis, or "marking," 3 for $\Sigma$, we can choose specific curves representing each homology class and all intersecting at one point $P$. See Fig. $2 a$. It is then useful to introduce the "cut" surface $\Sigma_{c}$ with the topology of a disk (Fig. 2b). Since $\Sigma_{c}$ involves more choices than just $A$, we will have to verify that constructions made with its help are unchanged as we vary the curves in their homology classes.

3 This is different from the sense of the word used in [36]. 
One useful calculation with $\Sigma_{c}$ is the following: let $\theta, \eta$ be closed 1-forms on $\Sigma$. Then $\int_{\Sigma} \theta \wedge \eta=\int_{\Sigma_{c}} \theta \wedge \eta=\int_{\Sigma_{c}} \mathrm{~d}(\varphi \eta)$ where $\theta=\mathrm{d} \varphi$ on $\Sigma_{c}$. By Stokes' theorem this is the integral of $\varphi \eta$ around the boundary of $\Sigma_{c}$. Grouping the boundary segments in pairs we get [37]

$$
\begin{aligned}
\int_{\Sigma} \theta \wedge \eta & =\sum_{i}\left[\varphi\left(a_{i}^{+}\right)-\varphi\left(a_{i}^{-}\right)\right] \oint_{b_{i}} \eta-\sum_{i}\left[\varphi\left(b_{i}^{+}\right)-\varphi\left(b_{i}^{-}\right)\right] \oint_{a_{i}} \eta \\
& =\sum_{i} \oint_{a_{i}} \mathrm{~d} \varphi \cdot \oint_{b_{i}} \eta-(a \leftrightarrow b),
\end{aligned}
$$

or

$$
\begin{aligned}
\int_{\Sigma} \theta \wedge \eta & =\sum\left[\oint_{a_{i}} \theta \oint_{b_{i}} \eta-\oint_{b_{i}} \theta \oint_{a_{i}} \eta\right] \\
& =(n, m) J\left(\begin{array}{c}
n^{\prime} \\
m^{\prime}
\end{array}\right)
\end{aligned}
$$

where we expanded $\theta, \eta$ in terms of $(n, m)$ and $\left(n^{\prime}, m^{\prime}\right)$. This identity is clearly independent of all choices made.

\section{B. Riemann Surfaces, Sheaves, and Bundles}

In order to define a Laplacian, say, we must have a metric. For many purposes, however, the conformal class of a metric is all we need. For example, given a metric class the Hodge theorem gives representative differential forms for the cohomology classes $\vec{\alpha}, \vec{\beta}$ dual to $A$, namely the harmonic forms. Also as is well known a conformal class amounts to a complex structure on $\Sigma[16][29]$. From now on $\Sigma$ will denote a surface endowed with such a structure, which is called a Riemann surface.

The complex coordinate patching functions of $\Sigma$ are analytic on the overlaps $U_{i} \cap U_{j}$ of coordinate charts. One can also consider holomorphic line bundles over $\Sigma$, complex bundles whose transition functions are always analytic. Such bundles are important because they have a well-defined notion of a holomorphic section. In fact there is a CauchyRiemann $\bar{\partial}$ operator on the sections of any holomorphic line bundle $\xi$, which we call $\bar{\partial}_{\xi}$; a holomorphic section $\sigma$ satisfies $\bar{\partial}_{\xi} \sigma=0$ (see sect. 5.C). Clearly, the derivatives of the patching functions define such a bundle, the holomorphic tangent $K^{-1}$ of $\Sigma$. Its dual $K$ is called the canonical bundle over $\Sigma$. We will always consider holomorphic bundles unless otherwise noted. 
Thus the bundles $K^{n}$ correspond to tensors with $n$ lower $z$ indices where $z$ is a local complex coordinate of $\Sigma$. Sections of $K^{n} \otimes \bar{K}^{m}$ are called $(n, m)$-tensors. In order to deal with spin we also define a holomorphic spin bundle ${ }^{4} L$ as any bundle such that $L \otimes L \cong K$; the corresponding tensors then have "half a $z$ index." Before describing these in greater detail, however, we first recall the notion of a sheaf [39].

The language of sheaves is useful for many constructions in geometry. We will only make essential use of it in the last section, however, and the reader may wish to skip the following paragraphs. While we will work on a surface $\Sigma$ in this section, most of the constructions have analogs in higher dimensions as well.

A sheaf $\mathcal{F}$ of abelian groups on $\Sigma$ is an assignment of an abelian group $\mathcal{F}(U)$ to every open set $U \subseteq \Sigma$. This is the only kind of sheaf we will consider in this paper. $\mathcal{F}(U)$ is called the group of "sections of $\mathcal{F}$ over $U . " \mathcal{F}$ also assigns to pairs of nested sets $V \subseteq U$ a "restriction" map $r_{U, V}: \mathcal{F}(U) \rightarrow \mathcal{F}(V)$ in a way which makes sense on overlaps. That is,

a) If $W \subseteq V \subseteq U$, then $r_{U, W}=r_{U, V} \circ r_{V, W}$.

b) If $\sigma_{1}, \sigma_{2}, \ldots$ are sections over $U_{1}, U_{2}, \ldots$, respectively and each pair $\sigma_{i}, \sigma_{j}$ have the same restriction to $U_{i} \cap U_{j}$, then each $\sigma_{i}$ is the restriction of some section $\rho$ over $U_{1} \cup U_{2} \cup \ldots$.

c) If $\rho$ is a section of $\mathcal{F}$ over $U \cup V$ which gives the identity when restricted to both $U$ and $V$, then $\rho$ is the identity.

Here are some examples of sheaves which we will use.

a) 0 . Over $U \subseteq \Sigma, O(U)=\{$ analytic functions on $U\}$; the group law is addition.

b) $\mathcal{M}$. Here $\mathcal{M}(U)=\{$ meromorphic functions on $U\}$. In between $\mathcal{O}$ and $\mathcal{M}$ we have

c) $O(P)$. If $P \in U$ then $O(P)(U)=$ functions analytic on $U$ except for a possible pole of first order at $P$ \}; otherwise $O(P)(U)=O(U)$. Note that the constant 1 is a canonical section of $O(U)$ over any $U$.

d) $O(-P)$. If $P \in U$ then $O(-P)(U)=$ functions analytic and vanishing to at least first order at $P\}$. Note that $O(-P)(\Sigma)$ has only the zero section, since

4 See [38], [29] for why this definition coincides with the usual definition of spin structure. 
$\Sigma$ is compact.

e) Given any holomorphic bundle $\xi$ on $\Sigma$, we can define analogously to (a) $\xi(U)=$ analytic sections of $\xi$ on $U$. We will not distinguish notationally between a bundle and its sheaf of holomorphic sections.

f) Given a vector space $\mathcal{V}$ and a point $P \in \Sigma$ we can let $\mathcal{F}(U)=\mathcal{V}$, if $P \in U$, and otherwise the zero vector space. The restriction map is either the identity or else the zero map. $₹$ is called the "skyscraper sheaf" and is denoted by $\left.\mathcal{V}\right|_{P}$. In particular, given a bundle $\xi$ and a point, we will write $\left.\xi\right|_{P}$ to denote both the fiber at $P$ and the corresponding sheaf with support at $P$.

g) Given any group such as $Z$, $R$, or $C$, we can define $Z(U)=Z$ etc. for every connected $U$. Every restriction is the identity. Sections of $Z$ etc. can be thought of as locally constant functions on $U$.

h) Finally, we can define sheaves where the group law is multiplication, not addition, of functions. These include the constant sheaf $\mathrm{C}^{*}=\mathrm{C}-\{0\}$ and the sheaf $\mathrm{O}^{*}$ whose sections are the local analytic functions which never vanish.

Thus roughly speaking the notion of sheaf generalizes that of bundle to include cases where the fiber dimension jumps (example $f$ ), as well as cases where only constant local sections are allowed (example $\mathrm{g}$ ).

In fact $O(P)$ is the sheaf of analytic sections of a certain bundle $\xi$, which we construct as follows. Let $U_{0}=\Sigma-\{P\}$ and $U_{1}$ a small disk neighborhood of $P$. Trivialize $\xi$ so that a section $s$ is given by functions $s_{i}$ on $U_{i}$ with $s_{1}=z \cdot s_{0}$ on $U_{0} \cap U_{1}$, where $z$ is a complex coordinate centered at $P$. Given a function $f$ in $O(P)(U)$ we get a section of $\xi$ on $U \cap U_{0}$, which we then analytically continue to $U \cap U_{1}$ using the transition function. Then clearly the functions in $O(P)(U)$ all correspond to smooth analytic sections of $\xi$. In particular, the canonical section 1 vanishes once at $P$. We will write $O(P)$ to refer either to the sheaf or the bundle, and $1_{O(P)}$ for the canonical section. Similarly $O(-P)$ gives a bundle via $s_{1}=z^{-1} \cdot s_{0}$. It too has a canonical section $1_{O(-P)}$ which now blows up at $P$.

A map between sheaves is a collection of homomorphisms $f_{U}: \mathcal{F}(U) \rightarrow \mathcal{G}(U)$ commuting with restriction. Roughly speaking, a sequence of maps is called exact at $P \in \Sigma$ 


$$
\cdots \rightarrow \mathcal{F}(U) \rightarrow \mathcal{G}(U) \rightarrow \cdots
$$

is exact for all sufficiently small neighborhood $U$ of $P$. (See [39] for the precise definition.) An exact sequence of the form

$$
0 \rightarrow \mathcal{E} \stackrel{\alpha}{\hookrightarrow} \mathcal{\beta} \stackrel{\beta}{\rightarrow} \mathcal{G} \rightarrow 0
$$

is called "short." Recall that this implies that $\alpha$ has no kernel and $\beta$ is onto, as well as that image $\alpha=\operatorname{ker} \beta$, again on small enough neighborhoods of each point of $\Sigma$. We will use three simple exact sequences:

a)

$$
0 \rightarrow 2 \pi i Z \stackrel{i}{\hookrightarrow} O \stackrel{\exp }{\longrightarrow} O^{*} \rightarrow 0
$$

Here $i$ is inclusion: every constant function is in particular an analytic function. Since $e^{2 \pi i x}=1$ iff $x$ is an integer, this sequence is exact.

b)

$$
\left.0 \rightarrow \xi \otimes O(-P) \stackrel{i}{\rightarrow} \xi \stackrel{r}{\rightarrow} \xi\right|_{P} \rightarrow 0
$$

where $\xi$ is any holomorphic bundle. Again $i$ includes the sections of $\xi$ vanishing at $P$ into all sections. $r$ restricts a section to its value at $P$, so the sequence is exact.

c)

$$
0 \rightarrow K \stackrel{i}{\longrightarrow} K \otimes O(P) \stackrel{\text { res }}{\longrightarrow} \mathrm{C}_{P} \rightarrow 0
$$

where $K$ is the cotangent bundle. $i$ includes the holomorphic sections into the ones holomorphic except for a possible first-order pole at $P$. res is the residue map: if $f$ is analytic at $P, \operatorname{res}_{P}\left(\frac{f}{z} \mathrm{~d} z\right)=f(P)$ is coordinate-invariant.

We can build a cohomology theory for any sheaf $F$ as follows [39][35][40].

We first define the groups of cochains with values in $\mathcal{F}$. Given a cover of $\Sigma$ by open sets $\left\{U_{\alpha}\right\}$, a 0 -cochain $\sigma \in C^{0}(\mathcal{F})$ is given by associating a section $\sigma_{\alpha} \in \mathcal{F}\left(U_{\alpha}\right)$ to every $U_{\alpha}$. The full cochain group is freely generated by such $\sigma$. A 1-cochain $\sigma \in C^{1}(\Sigma ; ₹)$ is given by associating a section $\sigma_{\alpha \beta} \in \mathcal{F}\left(U_{\alpha} \cap U_{\beta}\right)$ for every nonempty intersection, and so on. Next, introduce the coboundary operator 


$$
\begin{gathered}
\delta: C^{p} \rightarrow C^{p+1} \\
\delta \sigma\left(U_{0}, U_{1}, \ldots, U_{p+1}\right)=\left.\sum_{k=0}^{p+1}(-1)^{k} \sigma\left(U_{0}, \ldots, \hat{U}_{k}, \ldots, U_{p+1}\right)\right|_{U_{0} \cap \ldots \cap U_{p+1}}
\end{gathered}
$$

( $\hat{U}_{k}$ means that the $k+1$-entry is deleted.) It is easy to check that $\delta^{2}=0$. If $\sigma$ is a $p$-cochain satisfying $\delta \sigma=0$, we say that $\sigma$ is a cocyle. If $\sigma_{p}=\delta \sigma_{p-1}^{\prime}$ for some $(p-1)$-cochain $\sigma_{p-1}^{\prime}$, then we say that $\sigma_{p}$ is a coboundary. The $p$-th Cech cohomology group associated to the covering $\left\{U_{\alpha}\right\}$ is defined on the group of p-cocyles $Z^{p}$ modulo p-coboundaries $\delta C^{p-1}$ :

$$
H^{p}\left(\left\{U_{\alpha}\right\} ; \mathcal{F}\right)=Z^{p}\left(\left\{U_{\alpha}\right\} ; \mathcal{F}\right) / \delta C^{p-1}\left(\left\{U_{\alpha}\right\} ; \mathcal{F}\right)
$$

It is possible to define the cohomology groups $H^{p}(\Sigma ; \mathcal{F})$ as the "limit" of these groups as the covering $\left\{U_{\alpha}\right\}$ gets finer and finer [41]. For the constant sheaves of type $(g)$ above these groups are just the usual cohomology groups [41]. For the sheaf associated to a bundle $\xi$ we have that $H^{\circ}(\Sigma ; \xi)$ is just the space of global holomorphic sections, or in other words that

$$
H^{0}(\Sigma ; \xi)=\operatorname{ker} \bar{\partial}_{\xi}
$$

The other groups $H^{p}(\Sigma ; \xi)$ are more complicated.

Given a short exact sequence of sheaves (2.6), there is associated a long exact sequence of cohomology groups [41]:

$$
\begin{aligned}
0 & \rightarrow H^{0}(\Sigma ; \mathcal{E}) \rightarrow H^{0}(\Sigma ; \mathcal{F}) \rightarrow H^{0}(\Sigma ; \mathcal{G}) \rightarrow \\
& \rightarrow H^{1}(\Sigma ; \mathcal{E}) \rightarrow H^{1}(\Sigma ; \mathcal{F}) \rightarrow H^{1}(\Sigma ; \mathcal{G}) \rightarrow \cdots \\
\cdots & \rightarrow H^{p}(\Sigma ; \mathcal{E}) \rightarrow H^{p}(\Sigma ; \mathcal{F}) \rightarrow H^{p}(\Sigma ; \mathcal{G}) \rightarrow \cdots
\end{aligned}
$$

Using that $\alpha$ and $\beta$ in (2.6) commute with the coboundary operator $\delta$, it is easy to understand how one moves horizontally in the sequence (2.11). The step from $H^{p}(\Sigma ; \mathcal{G})$ to $H^{p+1}(\Sigma ; \mathcal{E})$ is more elaborate. Let $\sigma$ be a p-cocyle in $H^{p}(\Sigma ; \mathcal{G})$. We can represent $\sigma$ by a cocycle in $C^{p}\left(\left\{U_{\alpha}\right\} ; \mathcal{G}\right)$ for some covering of $\Sigma$. By exactness of $(2.6)$ at $\mathcal{G}$, we can find some $p$-cocycle $\tau$ in $C^{p}\left(\left\{U_{\alpha}^{\prime}\right\} ; \mathcal{F}\right)$ such that $\beta(\tau)=\sigma$, where $\left\{U_{\alpha}^{\prime}\right\}$ is some covering on $\Sigma$ finer than $\left\{U_{\alpha}\right\}$. Since $\delta(\sigma)=0$, and $\beta$ commutes with $\delta$, by exactness of (2.6) at $\mathcal{E}$ and $\mathcal{f}$ there exists a unique $(p+1)$-cocycle $\mu \in C^{p+1}\left(\left\{U_{\alpha}^{\prime}\right\} ; E\right)$ such that 
$\alpha(\mu)=\delta(\tau)$. The coboundary operator $\delta$ associates the class of $\mu$ to the class of $\sigma$. It is well-defined and independent of the choices made [41].

As a first application of sheaves we note that the transition functions of a holomorphic line bundle $\xi$ are analytic functions on patch overlaps, i.e., a chain ${ }^{5} t_{\alpha \beta}$ in $C^{1}\left(\Sigma ; 0^{*}\right)$. Moreover, the cocyle condition says that $(\delta t)_{\alpha \beta \gamma} \equiv 0$. Also redefining the local trivializations of $\xi$ gives an equivalent set of transition functions $t_{\alpha \beta}^{\prime}=t_{\alpha \beta} \cdot(\delta \nu)_{\alpha \beta}$, so that isomorphism classes of bundles are given by the group

$$
\operatorname{Pic}(\Sigma) \equiv H^{1}\left(\Sigma ; O^{*}\right)
$$

Pic $\Sigma$ is called the Picard group. Multiplication of transition functions $t_{\alpha \beta} \cdot \tilde{t}_{\alpha \beta}$ gives a new line bundle, the tensor product $\xi \otimes \tilde{\xi}$, while inversion $t_{\alpha \beta}^{-1}$ gives the dual bundle $\xi^{-1}$.

We can learn more about Pic $\Sigma$ by using the long exact sequence associated to (2.7):

$$
\begin{aligned}
\cdots H^{0}(\Sigma ; 0) & \stackrel{\exp }{\longrightarrow} H^{0}\left(\Sigma ; O^{*}\right) \stackrel{0}{\rightarrow} H^{1}(\Sigma ; 2 \pi i Z) \rightarrow H^{1}(\Sigma ; 0) \rightarrow H^{1}\left(\Sigma ; O^{*}\right) \\
& \stackrel{\operatorname{deg}}{\longrightarrow} H^{2}(\Sigma ; Z) \rightarrow H^{2}(\Sigma ; 0) \cdots
\end{aligned}
$$

Since the first $\exp : \mathrm{C} \rightarrow \mathrm{C}^{*}$ is onto, the next map must be zero. Also $H^{2}(\Sigma ; 0)$ is always zero, since by the Dolbeault theorem [39] it is isomorphic to $H_{\partial}^{0,2}(\Sigma)$ and there are no (0,2)-forms: $\mathrm{d} \bar{z} \wedge \mathrm{d} \bar{z}=0$. Hence we get (dropping the normalization $2 \pi i$ )

$$
0 \rightarrow H^{1}(\Sigma ; 0) / H^{1}(\Sigma ; Z) \rightarrow \operatorname{Pic}(\Sigma) \stackrel{\operatorname{deg}}{\rightarrow} H^{2}(\Sigma ; Z) \rightarrow 0
$$

Since $H^{2}(\Sigma ; Z)=Z$ we thus find that $\operatorname{Pic}(\Sigma)$ is disconnected, with identity component a Lie group we will call

$$
J(\Sigma) \equiv H^{1}(\Sigma ; 0) / H^{1}(\Sigma ; Z)
$$

Since $H^{1}(\Sigma ; 0)$ is a complex vector space, $J(\Sigma)$ is a complex space called the jacobian of $\Sigma$.

5 Here and in the sequel we are somewhat imprecise in our notation: We mean that we have chosen a covering $\left\{U_{i}\right\}$ for $\Sigma$ and used the transition functions to define a cochain in $C^{1}\left(\left\{U_{i}\right\} ; O^{*}\right)$. It turns out that different choices of covering lead to cohomologous $t_{\alpha \beta}$ 's in the limit of fine coverings mentioned above. 
The image $\operatorname{deg} \xi$ of a bundle in $Z$ is called the degree of $\xi$. Clearly $\operatorname{deg}\left(\xi_{1} \otimes \xi_{2}\right)=$ $\operatorname{deg} \xi_{1}+\operatorname{deg} \xi_{2}$, and indeed the degree is just the first Chern class of the $U(1)$ bundle associated to $\xi$. For example $O(P)$ has degree 1, as can easily be seen by working through the steps below (2.11). But the corresponding $U(1)$ bundle is the monopole, which has Chern number 1 as well. As another example the degree of the tangent bundle is the Euler number $\chi$, or

$$
\operatorname{deg} K=2(1-g)
$$

an even integer.

We can now return to the study of spin bundles. Suppose that $K$ is described by $g_{\alpha \beta} \in H^{1}\left(\Sigma ; O^{*}\right)$. We can construct a square root $L$ of $K$ by letting

$$
h_{\alpha \beta}= \pm \sqrt{g_{\alpha \beta}} \quad \text { on } \quad U_{\alpha} \cap U_{\beta} \text {. }
$$

Unfortunately, the cochain $h$ so defined will not in general be closed since

$$
\left(w_{2}\right)_{\alpha \beta \gamma} \equiv(\delta h)_{\alpha \beta \gamma}=h_{\beta \gamma} h_{\alpha \gamma}^{-1} h_{\alpha \beta}=h_{\alpha \beta} h_{\beta \gamma} h_{\gamma \alpha}
$$

can be \pm 1 on $U_{\alpha} \cap U_{\beta} \cap U_{\gamma}$. Hence an arbitrary choice of square roots in (2.13) will not generally define any bundle $L$.

Given a bad choice of $h_{\alpha \beta} \in C^{1}\left(\Sigma ; O^{*}\right)$, however, we can try to turn it into a good one by letting

$$
h_{\alpha \beta}^{\prime}=h_{\alpha \beta} \cdot f_{\alpha \beta} \quad \text { where } f \in C^{1}\left(\Sigma ; Z_{2}\right) \text {. }
$$

This changes $w_{2}$ to $w_{2}^{\prime}=w_{2} \cdot(\delta f)$. Hence if $w_{2}$ defines a trivial class in $H^{2}\left(\Sigma ; Z_{2}\right)$ then we can find an appropriate $f$ to shift it away and spin bundles will exist for $K$. But if we regard $\operatorname{deg} K$ as a class in $H^{2}(\Sigma ; Z)$, then working through the definitions shows that

$$
w_{2}=\exp (i \pi \cdot \operatorname{deg} K)
$$

Since the degree of $K$ is always even, $w_{2}$ is always trivial. Hence we can always arrange for $h$ to be closed: spin bundles always exist on any Riemann surface.

Now suppose that $h \in H^{1}\left(\Sigma ; O^{*}\right)$ describes a spin bundle $L$. Once again we can try modifying it by $f$ as in (2.14), where now $f$ must be closed. This will give a distinct new spin bundle whenever $f$ is not exact in $H^{1}\left(\Sigma ; Z_{2}\right)$. Thus the differences of spin bundles 
are given by $H^{1}\left(\Sigma, Z_{2}\right) \cong\left(Z_{2}\right)^{2 g}[35]$. Unfortunately there is no canonical, or preferred, spin structure on $\Sigma$, so we cannot directly parametrize all $L$ by $H^{1}\left(\Sigma, Z_{2}\right)$. Instead we will see that only after the introduction of a homology basis $A$ will there emerge a special $L_{A}$; we will then be able to describe other $L$ relative to this one.

Since $\operatorname{deg} K=2 g-2$, we find $\operatorname{deg} L=g-1$ for any $L$. We will use the term "twisted spin bundle" to refer to any $\xi$ of degree $g-1$, not necessarily satisfying $\xi^{2}=K$.

Before leaving sheaf theory we will describe without proof one more important theorem. This is Serre duality, which says that for any bundle $\xi$ we have

$$
H^{1}(\Sigma ; \xi) \cong\left[H^{0}\left(\Sigma ; K \otimes \xi^{-1}\right)\right]^{-1}
$$

As usual the inverse refers to the dual vector space. Also a simple argument shows that

$$
H^{1}(\Sigma ; \xi) \simeq \operatorname{coker} \bar{\partial}_{\xi}
$$

(Compare (2.10).) Again one uses the Dolbeault theorem to see that $H^{1}(\Sigma ; \xi)=$ $H_{\partial}^{(0,1)}(\Sigma ; \xi)$. Since all $(0,1)$-forms are $\bar{\partial}$-closed, we have all $\xi$-valued $(0,1)$-forms modulo the $\bar{\partial}$-exact ones, which is just coker $\bar{\partial}_{\xi}$.

Taking $\xi$ to be trivial, (2.15) says $H^{1}(\Sigma ; 0) \cong\left[H^{\circ}(\Sigma ; K)\right]^{-1}$. The space $H^{\circ}(\Sigma ; K)$ of holomorphic 1-forms is always $g$-dimensional. One can see this by noting that if $\eta \in$ $H^{0}(\Sigma ; K)$ then the real and imaginary parts of $\eta$ are harmonic 1 -forms, and so can be expanded in the basis of $2 g$ real harmonic 1-forms $\alpha^{i}, \beta^{j}$ mentioned at the beginning of this section. The space of complex linear combinations of $\alpha, \beta$ with no $\bar{z}$ piece then has $g$ dimensions. Holomorphic 1-forms are also called "abelian differentials."

Thus the identity component of $\operatorname{Pic}(\Sigma)$ is $J(\Sigma) \cong\left[H^{\circ}(\Sigma ; K)\right]^{-1} / H^{1}(\Sigma ; Z)$, a torus of $g$ complex dimensions. Hence it is compact, as promised. We have seen how it parametrizes degree-zero bundles, or differences of degree-d bundles for any $d$.

\section{Divisors}

In our discussion of sheaves we came across bundles we called $O(P)$ and $O(-P)$. It will be useful to generalize these by introducing the notion of a divisor; divisors give a second description of holomorphic line bundles.

A divisor is a formal linear combination of points of $\Sigma$ with signed multiplicities: $D=\sum_{i} n_{i} P_{i}$. To such a $D$ we associate the line bundle $O(D) \equiv \bigotimes_{i} O\left(P_{i}\right)^{n_{i}} . O(D)$ 
is perfectly regular at the points $P_{i}$, but it comes equipped with a section $1_{O(D)} \equiv$ $\bigotimes_{i}\left(1_{O\left(P_{i}\right)}\right)^{n_{i}}$ which has zeros (resp. poles) at those $P_{i}$ with $n_{i}>0$ (resp. $n_{i}<0$ ). We can add divisors in the obvious way, whereupon the map $\tilde{I}: D \mapsto O(D)$ becomes a homomorphism. Under $\tilde{I}$ the degree of $O(D)$ equals $\sum n_{i}$, as we have noted.

Conversely, given any bundle $\xi$ we can find a divisor as follows. Every $\xi$ has many meromorphic sections [42]. Choose any one section $s$ and let $\operatorname{div}(s)=\sum n_{i} P_{i}-\sum m_{j} Q_{j}$, where $\left\{P_{i}\right\}$ are the zeros of $s$ of order $n_{i}$ and $\left\{Q_{i}\right\}$ are poles of order $m_{j}$. This map inverts $I$, but it is ambiguous: letting $s^{\prime}=f \cdot s$ changes $\operatorname{div}(s)$ by the divisor of any meromorphic function $f \in M(\Sigma)$. Thus we define the group of divisor classes as all divisors modulo the divisors of meromorphic functions, to get

$$
I:\{\text { divisor classes }\} \rightarrow \operatorname{Pic}(\Sigma)
$$

To show that $I$ is an isomorphism we further remark that a nontrivial bundle is never represented by the zero divisor. If it were then the meromorphic section $s$ with no zeros or poles would trivialize $\xi$.

Note that while two divisors $D \neq D^{\prime}$ may give rise to isomorphic bundles, still the canonical sections $1_{O(D)}, 1_{O\left(D^{\prime}\right)}$ are totally different: they vanish and blow up at different places and so cannot correspond to each other under the isomorphism $O(D) \cong O\left(D^{\prime}\right)$. Conversely, a given bundle has no canonical meromorphic section; only after a specific divisor has been chosen in its class is such a section available.

From now on we will not distinguish notationally between divisor classes and bundles. That is, we will sometimes drop $I$ (and $\tilde{I}$ ) from formulas.

Since (2.17) is an isomorphism we can represent any bundle as any other one times some $O(D)$. We can use this fact to extend a simple result about the cotangent $K$ to arbitrary bundles. Note that $H^{\circ}(\Sigma ; 0) \cong C$ since the only analytic functions are the constants, while $\operatorname{dim} H^{0}(\Sigma ; K)=g$ as mentioned earlier. Hence $\operatorname{dim} H^{0}(\Sigma ; 0)-$ $\operatorname{dim} H^{\circ}(\Sigma ; K)=\operatorname{deg} O+1-g$ since $\operatorname{deg} O=0$. Now consider the corresponding statement for arbitrary $\xi$ :

$$
\operatorname{dim} H^{\circ}(\Sigma ; \xi)-\operatorname{dim} H^{0}\left(\Sigma ; K \otimes \xi^{-1}\right)=\operatorname{deg} \xi+1-g
$$

To derive (2.18) from the preceding version express $\xi$ as $\xi \cong K \otimes O(D)$ for some $D$. The 
difference between the two equations can be shown to hold by repeated application of the long exact sequence associated to (2.8). (2.18) together with (2.15) gives the classical Riemann-Roch theorem.

In the remainder of this section we will make the isomorphism (2.17) explicit with the help of a homology basis $A$. That is, we will define a map $I_{A}$ from divisor classes to a complex torus $J_{A}$. While the constructions are not intrinsic, they are helpful for making the connection to theta functions.

We can choose a basis of $H^{0}(\Sigma ; K)$, or abelian differentials, by requiring that

$$
\int_{a_{i}} \omega_{A}^{j}=\delta_{i j} .
$$

It is then useful to define the "period matrix"

$$
\left[\tau_{A}\right]^{i j} \equiv \int_{b_{i}} \omega_{A}^{j} .
$$

$\tau_{\mathcal{A}}$ is useful because it characterizes the surface $\Sigma$. Indeed Torelli's theorem implies that if two marked Riemann surfaces $\Sigma$ and $\Sigma^{\prime}$ of the same genus have the same period matrix then they are isomorphic as Riemann surfaces, although the converse is certainly false $\left(\tau_{\mathcal{A}} \neq \tau_{\widetilde{A}}\right.$ in general for the same surface $\Sigma$ with two markings). Using (2.5), $\tau_{\mathcal{A}}$ is easily seen to be symmetric with positive definite imaginary part [37][6][29]. Thus we can define

$$
Y_{A}=\left(\tau_{A}-\bar{\tau}_{A}\right)^{-1}
$$

Note that $\left(Y_{A}\right)_{i j}^{-1}=\int_{\Sigma} \bar{\omega}^{i} \wedge \omega^{j}$ by (2.5), (2.19), and (2.20).

It is useful to know how things change when we change the marking $A$. If $\tilde{A}=A \Lambda^{-1}$ is a new basis as in (2.2), then the definitions give

$$
\begin{gathered}
\vec{\omega}_{\tilde{A}}=\vec{\omega}_{\mathcal{A}} \cdot\left(C \tau_{A}+D\right)^{-1}, \\
\tau_{\tilde{A}}=\left(A \tau_{A}+B\right)\left(C \tau_{A}+D\right)^{-1}, \text { and } \\
Y_{\widetilde{A}}=\left(C \tau_{\mathcal{A}}+D\right) \cdot Y_{A} \cdot\left(\overline{C \tau_{A}+D}\right)^{t} .
\end{gathered}
$$

We will sometimes drop the subscript $A$ when it is clear which homology basis is meant.

Given a marked surface $\Sigma_{\mathscr{A}}$ we can now build a complex $g$-torus:

$$
\begin{aligned}
& J_{A}=C^{g} / \Gamma_{A} ; \\
& \Gamma_{A}=Z^{g} \oplus \tau_{A} \cdot Z^{g} .
\end{aligned}
$$


Changing homology bases as in (2.2), then we get a map from $J_{A} \rightarrow J_{\tilde{A}}$ which sends $\vec{z} \in \mathrm{Z}^{g}$ to

$$
\widetilde{z}=z \cdot\left(C \tau_{A}+D\right)^{-1}
$$

We can now define a map $I_{A}$ from divisor classes of degree zero to $J_{A}$, commuting with the canonical identifications (2.25). Note that a divisor $D$ of degree zero is the boundary $\partial c$ of some 1-cochain obtained by "connecting the dots." Let

$$
\tilde{I}_{A}^{i}[D]=\int_{C} \omega_{A}^{i} \quad \bmod \Gamma_{A}
$$

Using (2.22), it is clear that (2.26) commutes with (2.25). Also, if we change $\sigma$ to $\sigma^{\prime}$, then $\sigma-\sigma^{\prime}$ is a cycle and so $\tilde{I}_{A}[D]$ is unchanged modulo $\Gamma_{A}$, by (2.19) and (2.20).

Finally $\tilde{I}_{\mathcal{A}}$ defines a map $I_{\mathcal{A}}$ on divisor classes. That is $\tilde{I}_{A}[D]=0$ if and only if $D=\operatorname{div} f$ for some meromorphic function $f$. This is called Abel's theorem. Thus $I_{\mathcal{A}}$ sets up an isomorphism between the abstract jacobian $J(\Sigma)$ and its concrete version $J_{A}$.

\section{Curvature and Holonomy}

There is one final characterization of holomorphic line bundles which we will use, involving holonomy. It is also time to begin introducing metrics and hence geometry on our bundles.

Let $\xi$ be a holomorphic line bundle with a smooth hermitian norm $\|\cdot\|$. We can describe sections of $\xi$ relative to one local trivializing sections $s$ as $\sigma=f \cdot s$, where $f$ is some function. "Trivializing" means that in some open set $s$ is analytic and nonvanishing. With respect to the frame $s$ we can now write down a covariant derivative [43]:

$$
\begin{aligned}
& \bar{D} \sigma=(\bar{\partial} f) s \\
& D \sigma=(\partial f+\Theta f) s,
\end{aligned}
$$

where

$$
\Theta=\partial \log \|s\|^{2}
$$

is the connection 1-form. The corresponding curvature is

$$
R=\bar{\partial} \Theta=\bar{\partial} \partial \log \|s\|^{2}
$$


and it is independent of the trivializing section $s$ chosen. If $s$ instead vanishes or has a pole somewhere, then (2.27) must be modified to remove the resulting delta-function singularities.

The Chern-Weil construction represents the Chern number in terms of the integral of $R$. In fact we can again see that this number equals the degree since

$$
\int_{\Sigma} R=-2 \pi i \operatorname{deg} \xi
$$

To prove (2.28), let $\xi=O(P), \quad s=1_{O(P)}$, and integrate over $\Sigma$ minus a small neighborhood $U$ of $P$. Since $R=\mathrm{d}\left(\partial \log \left\|1_{O(P)}\right\|^{2}\right)$ away from $P$ we get $-\oint_{\partial U}\left(\partial_{z} \log \left\|1_{O(P)}\right\|^{2}\right) \cdot \mathrm{d} z$. But $1_{O(P)}$ is vanishing linearly near $p$, so in any smooth metric this equals $-\oint \frac{\mathrm{d} z}{z}=-2 \pi i$. The reader may want to work out (2.28) explicitly on the sphere with $\xi=K$ to recover $\operatorname{deg} K=-2$.

We now consider a closed curve $\gamma: S^{1} \rightarrow \Sigma$. Given a vector $\left.v \in \xi\right|_{\gamma(0)}$ we can transport $v$ around $\gamma$ while preserving the relation:

$$
\langle D v, \dot{\gamma}\rangle=0
$$

Then $v(1)$ will again live in $\left.\xi\right|_{\gamma(0)}$, and we can let

$$
v(1)=v(0) \mathrm{e}^{2 \pi i H(\gamma ; \xi)}
$$

define the holonomy of $\xi$, with its given metric, around $\gamma$. Supposing that $\gamma$ lies entirely in one trivializing patch, it is easy to show that

$$
H(\gamma ; \xi)=-\frac{1}{2 \pi i} \int_{S^{1}} \gamma^{*} \Theta \quad \bmod \mathrm{Z}
$$

Further manipulation shows that $\mathrm{e}^{2 \pi i H}$ is well-defined under change of trivialization and $H$ is real.

The holonomy changes in a simple way when we deform $\gamma$ into a nearby $\gamma^{\prime}$ (Fig. 3 ). Suppose $\delta$ is the region of $\Sigma$ lying between $\gamma$ and $\gamma^{\prime}$, with orientation given by $(\dot{\gamma}, \mathbf{n})$, where $n$ is the outward normal at $\gamma$. Then $R=\mathrm{d} \Theta$ and Stoke's theorem say that

$$
H(\widetilde{\gamma}, \xi)-H(\gamma, \xi)=-\frac{1}{2 \pi i} \int_{\delta} R
$$




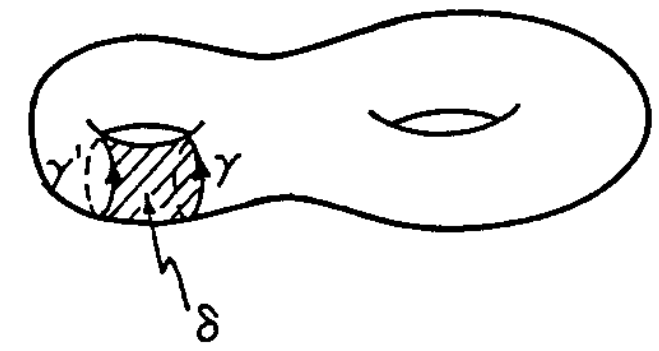

Fig. 3: Deforming a cut on $\Sigma$.

In particular if $R \equiv 0$, so that $\operatorname{deg} \xi=0$, then $H$ depends only on the homology class of $\gamma$. It therefore defines a real cohomology class, modulo an integral class, which we will call $H(\xi) \in H^{\mathrm{l}}(\Sigma ; \mathrm{R}) / H^{1}(\Sigma ; \mathrm{Z})$.

Actually every degree-zero bundle admits a flat metric, which is unique up to a constant. To see this we choose an arbitrary norm with curvature $R, \int R=0$, and modify the norm by a nonvanishing function $\exp (h)$. Then $h$ should satisfy $\bar{\partial} \partial h=-R$, which can be solved since the right side is orthogonal to the constant function. Thus $H(\xi)$ depends only on $\xi$.

The flat holonomy $H(\xi)$ vanishes if and only if $\xi$ is trivial. For, if $\xi$ is flat we can let $s$ be a holomorphic, covariantly constant section throughout the cut Riemann surface $\Sigma_{c}$. If $H(\xi)=0$ then $s$ does not jump across the cuts and $\xi$ is trivial, whereas if $\xi$ is trivial we can consider the flat metric as a real function satisfying $\bar{\partial} \partial h=0$, or $h=$ constant; then $\Theta=0$ and $H=0$. Furthermore every $H \in H^{1}(\Sigma ; \mathrm{R}) / H^{1}(\Sigma ; \mathrm{Z})$ actually arises as $H(\xi)$ for some $\xi: \xi$ is just the bundle with constant transition functions $\exp \left(-2 \pi i\left\langle H, a_{i}\right\rangle\right), \exp \left(-2 \pi i\left\langle H, b_{i}\right\rangle\right)$ across the cuts.

We thus have three intrinsic ways of describing degree-zero holomorphic line bundles: as patching data (i.e. $J(\Sigma)$ ), as divisor classes, and as real 1-forms defined modulo integers. In addition we have a description in terms of a homology basis (namely $J_{A}$ ) and maps $I, I_{\mathscr{A}}$, and $H$ making all the viewpoints isomorphic. See Fig. 4, where we have for convenience defined three more maps by requiring that everything commute.

Of the new maps in Fig. 4 it will be useful to know $F_{A}$ explicitly. This takes 


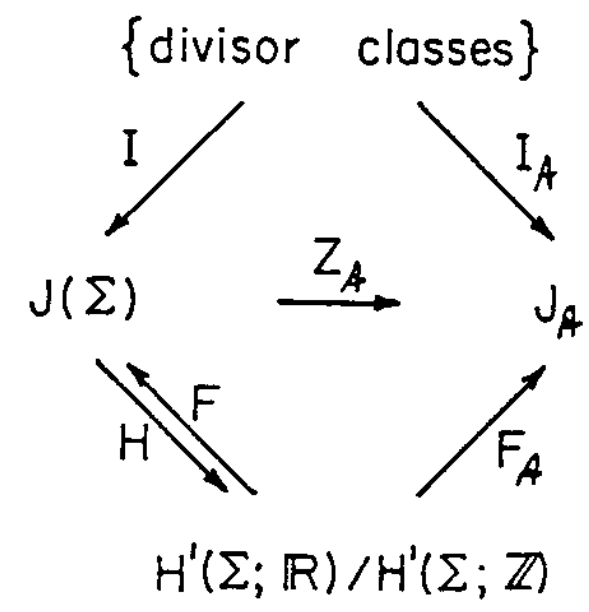

Fig. 4: Three ways to describe flat line bundles on a Riemann surface.

holonomy data and translates it into a point in $C^{g}$ defined modulo $\Gamma_{\mathscr{A}}$ :

$$
F_{A}: \psi \mapsto-\left(1, \tau_{A}\right) J\left(\begin{array}{l}
p \\
q
\end{array}\right)
$$

where

$$
\psi=(\vec{\alpha}, \vec{\beta})\left(\begin{array}{l}
\vec{p} \\
\vec{q}
\end{array}\right)
$$

To show that (2.32) is right one must find the holonomy of a flat bundle with a given divisor $D$, then show that $I_{A}[D]$ agrees with (2.32). This is done in Appendix A. Note that after a change of marking $\psi$ is represented by $(\underset{\widetilde{q}}{\widetilde{q}})=\left(\Lambda^{-1}\right)^{t}\left(\begin{array}{l}p \\ q\end{array}\right)$. Using the symplectic property $\Lambda^{t} J \Lambda=J$, one can show that (2.32) indeed commutes with the identification $J_{A} \cong J_{\widetilde{A}}$

Before closing this section we note that the complex torus $J(\Sigma)$ has a natural hermitian norm. In terms of $J_{A}$ this is

$$
B_{A}\left(z, z^{\prime}\right)=2 i \bar{z} \cdot Y_{A} \cdot z^{\prime}
$$

where $Y_{A}=\left(\tau_{A}-\bar{\tau}_{A}\right)^{-1}$. One can verify using (2.25), (2.24) that the $B_{A}$ define an intrinsic norm $B$ on $J(\Sigma)$. Note that one has

$$
\operatorname{Im} B\left(F(\psi), F\left(\psi^{\prime}\right)\right)=\int_{\Sigma} \psi \wedge \psi^{\prime}=(n, m) J\left(\begin{array}{c}
n^{\prime} \\
m^{\prime}
\end{array}\right)
$$




\section{E. Theta Functions and Spinors}

We will be brief in this subsection; see [37][6][29]. The Riemann theta function is defined by

$$
\vartheta(z \mid \tau)=\sum_{\vec{n} \in \mathbf{Z}^{\circ}} \exp (i \pi n \cdot \tau \cdot n+2 \pi i n \cdot z)
$$

and satisfies

$$
\vartheta(z+\tau \cdot n+m \mid \tau)=\mathrm{e}^{-i \pi n \cdot \tau \cdot n-2 \pi i n \cdot z} \vartheta(z \mid \tau)
$$

Thus if we insert a period matrix $\tau_{A}$ of some marked Riemann surface $\Sigma_{A}, \vartheta\left(z \mid \tau_{A}\right)$ defines a section of a holomorphic bundle over the torus $J_{\mathfrak{A}}$. This bundle is not trivial since $\vartheta$ sometimes vanishes but never blows up. The set $\Theta_{A} \subseteq J_{\mathcal{A}}$ where it vanishes has complex codimension one and so is the natural generalization of the notion of divisor to $g$ dimensions.

The component $\mathrm{Pic}_{g-1}$ of the Picard group consisting of bundles of degree $g-1$ also has a divisor of special points, called simply $\Theta$, the "theta divisor." These are the twisted spin bundles $\xi$ for which $\bar{\partial}_{\xi}$ has a zero mode, that is, the ones which admit a global holomorphic section. For example, if $\Sigma$ is the torus then $J(\Sigma)$ is itself a complex torus of dimension one. Thus we expect $\Theta$ to consist of discrete points, and indeed it is exactly one point: the trivial bundle is the only one of degree $g-1=0$ admitting a single-valued holomorphic section.

The Riemann vanishing theorem can be used to characterize the zeros of $\vartheta$. It implies [37][6][29] that for any homology basis $A$ there is a preferred spin bundle $L_{A}$ with the property that

$$
\Theta_{A}=\left\{I_{A}\left[L_{A} \otimes L^{-1}\right], \quad \text { as } L \text { runs through } \Theta\right\}
$$

(As mentioned, sometimes we will write a bundle for the corresponding divisor class.) That is, for fixed $\tau_{A} \vartheta\left(z \mid \tau_{A}\right)$ vanishes precisely on a set which is $\Theta$ shifted by the preferred spin bundle $L_{A} \cdot{ }^{6}$

We can use (2.37) to parametrize all twisted spin bundles given a marking, as $L=$ $L_{\mathfrak{A}} \otimes F_{A}(\psi)$ where $\psi=(\alpha, \beta)\left(\begin{array}{l}p \\ q\end{array}\right)$ is a real cohomology class and $F_{A}$ is as in Fig. 4 .

${ }^{6}$ If we choose a point $P_{0}$ on $\Sigma$ then $I_{A}\left[L_{A} \otimes O\left((1-g) P_{0}\right)\right]$ is a point in $J_{A}$ called the "vector of Riemann constants." 
When we change the marking, however, we must be careful to account for the fact that $L_{\tilde{\AA}} \not=L_{\AA}$. Instead, if

$$
\begin{gathered}
L=L_{\mathcal{A}} \otimes F_{A}(\psi)=L_{\tilde{A}} \otimes F_{\mathfrak{A}}(\hat{\psi}) \\
\psi=(\alpha, \beta) J\left(\begin{array}{l}
\phi \\
\theta
\end{array}\right) \quad \hat{\psi}=(\alpha, \beta) J\left(\begin{array}{c}
\hat{\phi} \\
\hat{\theta}
\end{array}\right)
\end{gathered}
$$

then (see (2.3))

$$
\left(\begin{array}{l}
\hat{\phi} \\
\hat{\theta}
\end{array}\right)=\Lambda\left(\begin{array}{l}
\phi \\
\theta
\end{array}\right)+\delta ; \quad \delta=\left[\begin{array}{c}
\frac{1}{2}\left(A B^{t}\right)_{d} \\
\frac{1}{2}\left(C D^{t}\right)_{d}
\end{array}\right] .
$$

The inhomogeneous term $\delta$ added to (2.4) represents the change from $L_{A}$ to $L_{\widetilde{A}}$. The subscript $d$ means the vector built from the diagonal elements of a matrix. The extra $J$ in the description of $\psi$ is a traditional notation for bundles. From now on $\left(\begin{array}{l}\phi \\ \theta\end{array}\right)$ will refer to $\psi$ as in $(2.39)$, while $(n, m)$ still refers to $(n, m)\left(\begin{array}{l}\alpha \\ \beta\end{array}\right)$.

Equation (2.40) can be derived from (2.37) and the fact that $\vartheta\left(\hat{\phi}+\tau_{\widetilde{A}} \hat{\theta} \mid \tau_{\widetilde{A}}\right)$ is a nonzero factor times $\vartheta\left(\phi+\tau_{\mathcal{A}} \theta \mid \tau_{\mathcal{A}}\right)$ [44]. More specifically, one shows that [44]

$$
\vartheta\left[\begin{array}{c}
\hat{\hat{\theta}} \\
\hat{\phi}
\end{array}\right]\left(0 \mid \tau_{\widetilde{A}}\right)=\epsilon \cdot \operatorname{det}\left(C \tau_{\mathcal{A}}+D\right)^{1 / 2} \cdot \vartheta\left[\begin{array}{l}
\theta \\
\phi
\end{array}\right]\left(0 \mid \tau_{\mathcal{A}}\right)
$$

where $\epsilon$ is a phase and the "theta function with characteristics" is a convenient modification of (2.35), defined as

$$
\begin{aligned}
\vartheta\left[\begin{array}{l}
\theta \\
\phi
\end{array}\right](z \mid \tau) & \equiv \sum_{\vec{n} \in \mathbf{Z}^{\theta}} \exp [i \pi(n+\theta) \tau(n+\theta)+2 \pi i(n+\theta)(z+\beta)] \\
& =\exp [i \pi \theta \cdot(\tau \cdot \theta+2(z+\phi))] \vartheta(z+\phi+\tau \theta \mid \tau) .
\end{aligned}
$$

If $\phi, \theta$ have half-integer entries, so that $L$ in (2.38) is a spin bundle, then the phase $\epsilon$ in (2.41) is always an eighth root of unity.

Equation (2.42) is also a useful modification of (2.35) in that its transformation analogous to (2.36) is by a pure phase. Thus its absolute square is an ordinary continuous real function on the torus $J_{\mathcal{A}}$. We will denote this function by

$$
\mathcal{N}_{A}(z)=\mathrm{e}^{-4 \pi i y \cdot Y \cdot y}|\vartheta(z \mid \tau)|^{2},
$$


where $y=\operatorname{Im} z . \mathcal{N}$ can be regarded as a metric on the bundle over $J_{A}$ defined by $\vartheta$, or more precisely,

$$
\mathcal{N}_{A}=\left\|1_{O\left(\Theta_{A}\right)}\right\|^{2}
$$

a fact we use in Appendix A.

\section{F. Arakelov Metrics}

If we are given a metric on the cotangent bundle $K$, then we get at once metrics on all powers of $K$, with the property that

$$
R_{K^{\lambda}}=\lambda R_{K}
$$

For fractional $\lambda$ this is independent of the spin structure chosen, since all spin structures differ by flat bundles. In this section we will describe a particularly useful metric first described by Arakelov [20] and used extensively by Faltings [19]. While we will explicitly show that our results remain true for every metric, the use of the Arakelov metric in the intermediate stages will simplify our formulas somewhat in sect. 5 .

Given an arbitrary metric $\|\cdot\|^{2}$ on $K$ we can define metrics on the bundles $O(P)$ as follows. Define the $(1,1)$-form

$$
\mu \equiv \frac{1}{4 \pi i(1-g)} R_{K}
$$

so that $\int \mu=1$. Define next an electrostatic Green function $\log G$ on $\Sigma$ by

$$
\partial_{P} \bar{\partial}_{P} \log G(P, Q)=i \pi\left(\mu(P)-\delta_{Q}(P)\right)
$$

where $\delta_{Q}$ is the delta function: $\int f \cdot \delta_{Q}=f(Q) . \delta_{Q}(P)$ is a $(1,1)$-form at $P$. The $\mu$ is needed in (2.45) so that the right side integrates to zero. Then $G$ equals $\left|z_{P}-z_{Q}\right|$ times a smooth function as $P \rightarrow Q$, and also $G(P, Q)=G(Q, P)[20]$. We will fix the normalization of $G$ by requiring

$$
\int \log G(P, Q) \cdot \mu(P)=O
$$

We will need to know how $G$ changes when we rescale the original metric on $K$. Suppose

$$
\tilde{g}^{z \bar{z}}=\mathrm{e}^{2 \sigma} g^{z \bar{z}}
$$


Then $\tilde{\mu}=\mu+[2 \pi i(1-g)]^{-1} \bar{\partial} \partial \sigma$ and we still have $\int \tilde{\mu}=1$. Working through the definitions one finds that $(2.45)$ implies $\tilde{G}(P, Q)$ is $\exp \left[\frac{1}{2(g-1)} \sigma(P)\right]$ times a function of $Q$ only. Requiring symmetry and the normalization condition (2.46), we get

$$
\tilde{G}(P, Q)=\exp \left[\frac{1}{4 \pi i(g-1)^{2}} S_{L}[\sigma]+\frac{1}{2(g-1)}(\sigma(P)+\sigma(Q))\right] \cdot G(P, Q)
$$

where the "Liouville action" is

$$
S_{L} \equiv \int_{\Sigma}(\partial \sigma \wedge \bar{\partial} \sigma+\sigma R)
$$

and $R$ is the original curvature.

Next, using $G$ we can put a norm on any $O(P)$ bundle. We declare that for the unit section,

$$
\left\|1_{O(P)}\right\|(Q)=G(P, Q)
$$

While the right-hand side vanishes at $P$, so does the section $1_{O(P)}$, so the norm defined by (2.50) is nonsingular. We can generalize (2.50) to an arbitrary bundle $O(D)$ by taking $O(p+q) \cong O(p) \otimes O(q)$ to be an isometry.

We can now define another norm on the cotangent $K$. Near any point $Q$, declare that the distance $d^{\prime}(P, Q)$ between $Q$ and a nearby point $P$ should approach $G(P, Q)$ as $P \rightarrow Q$, or in other words that

$$
\|\mathrm{d} z\|^{\prime}(Q)=\lim _{P \rightarrow Q} \frac{\left|z_{P}-z_{Q}\right|}{G(P, Q)} .
$$

It is easy to show that (2.51) defines a norm at $P$ which is independent of the choice of coordinate $z$. Indeed (2.51) just states that the residue map

$$
\left.[K \otimes O(P)]\right|_{P} \cong \mathrm{C}
$$

(see (2.9) ) should be an isometry in the new norm on $K$. The new norm $\|\cdot\|^{\prime}$ is perfectly smooth on $\Sigma$. However, it will not in general be simply related to the metric $\|$. $\|$ we started with.

Arakelov's norm is defined to be the one for which the above procedure reproduces the original metric on $K$. That is, if we take $\mu$ to be related to the Arakelov curvature by (2.44), then we get metrics on the $O(D)$ bundles such that (2.51) gives $\|\cdot\|=\|\cdot\|^{\prime}$. The Arakelov metric is unique, and its curvature is [20] 


$$
\mu_{\mathrm{Arak}}=\frac{1}{g} \bar{\omega}_{A}^{i} Y_{A}^{i j} \omega_{A}^{j} ; \quad Y_{A}=\left(\tau_{A}-\bar{\tau}_{A}\right)^{-1} .
$$

The corresponding curvature on the tensors of spin $\lambda$ is

$$
R_{K^{\lambda}}=4 \pi i(1-g) \lambda \mu_{\mathrm{Arak}}
$$

More generally, any bundle with metric curvature proportional to $\mu_{\text {Arak }}$ is said to have an "admissible metric." Since we will be using (2.51) repeatedly, the choice of admissible metrics will simplify many formulas. Specifically, (2.51) expresses the metric on $K$ in terms of the regulated coincident Green function of the bosonic theory.

We emphasize again that the choice of admissible metrics is for convenience only, and that bosonization works in any metric.

\section{The Bosonic Action}

In this section we will arrive at an action functional for a scalar theory which is to reproduce a general first-order fermionic system. We will begin by reviewing the situation on the sphere. Next we proceed to higher genus, first in spin-1/2 and then for general spin. Throughout, we will consider only free fermions, that is, fermions interacting with background gauge and gravitational fields but without self-interactions such as mass or quartic terms. This is the case of interest for the NSR superstring in flat spacetime.

We begin by reviewing existing results to fix notation. The prototypical fermionic system one might wish to bosonize has action

$$
S_{f}=\int_{\Sigma} \bar{\psi} i \not \partial \psi
$$

In euclidean path integrals $\psi$ and $\bar{\psi}$ are independent, and it has become traditional to rename the fields, with $\psi_{1} \mapsto c \quad \psi_{2} \mapsto \bar{b} \quad \bar{\psi}_{1} \mapsto b$ and $\bar{\psi}_{2} \mapsto \bar{c}$. Using complex notation and rescaling fields we then have

$$
S_{f}=\frac{i}{2 \pi} \int_{\Sigma}(b \bar{\partial} c+\bar{b} \partial \bar{c})
$$

where $b$ and $c$ are sections of a spin bundle $L$, and $\bar{b}$ and $\bar{c}$ are sections of $\bar{L}$. More generally we can let $c$ be a section of any holomorphic bundle $\xi$, and $b$ a section 
of $K \otimes \xi^{-1}$. In any case the integrand is a $(1,1)$-form and so can be integrated over $\Sigma$ without the use of any metric. That is, (3.1) is classically conformally invariant. It is also invariant under the global chiral transformation $b \mapsto \mathrm{e}^{i \alpha} b, c \mapsto \mathrm{e}^{-i \alpha} c$.

Similarly the prototypical scalar action for a single real field $\varphi$ has the form

$$
\begin{aligned}
S_{1} & =\int_{\Sigma} 2 \pi \vec{\nabla} \varphi \cdot \vec{\nabla} \varphi d(\mathrm{vol}) \\
& =\int_{\Sigma} 4 \pi i \partial \varphi \wedge \bar{\partial} \varphi .
\end{aligned}
$$

The unusual normalization is for later convenience. The second form makes it clear that $S_{1}$ is also classically conformally invariant. It also has an invariance under shifts $\varphi \mapsto \varphi+$ constant.

The operator analysis of bosonization on $2 d$ Minkowski spaces teaches us two important physical lessons. (See for example [1] [45] [46] [47] [25] [26].) First of all, the correspondence should be between the fermionic bilinears and exponentials of $\varphi$, properly normal-ordered. Secondly, the bosonic field $\varphi$ is properly to be regarded as a periodic, or circle-valued, field. This fact is compatible with the first if the normalization of $\varphi$ is chosen such that its ambiguity does not affect its exponential. We can see the periodicity of $\varphi$ either in the periodic sine-Gordon potential of $[1]$ or in the case where spacetime is the Minkowski cylinder [46]. In either case the crucial physical basis of bosonization is that fermions in (3.1) correspond to solitons, or states where $\varphi$ is multiple-valued, in (3.2). In particular, when spacetime has noncontractible loops the partition function of the bosonic system gets important contributions from soliton sectors.

Since we will use a covariant path integral and analyze surfaces with many noncontractible loops, we will sometimes use the term "instanton" to describe any field configuration $\varphi$ with nontrivial winding numbers in some direction. Clearly we have one independent winding number for every element of a homology basis (Fig. 1). Since these winding numbers are unaffected by a shift of $\varphi$ by a constant, they amount to specifying the cohomology class of the real 1-form $\mathrm{d} \varphi$.

\section{A. The Sphere}

On the sphere, the second observation above is immaterial, since there are no noncontractible loops on $\Sigma$ and hence no solitons. To make the first observation concrete in 
our present notation we will assume that we have

$$
\begin{gathered}
b \bar{b} \propto \mathrm{e}^{q \varphi} \\
c \bar{c} \propto \mathrm{e}^{-q \varphi}
\end{gathered}
$$

and find $q$, starting with the case of spin-1/2. First note that (3.2) gives a two-point function with singularity

$$
\langle\varphi(z) \varphi(w)\rangle \sim-\frac{1}{16 \pi^{2}} \log |z-w|^{2} .
$$

In proving this we have used the fact that

$$
\bar{\partial}_{P} \partial_{P} \log \left|z_{P}-z_{Q}\right|^{2}=2 \pi i \delta_{Q}(P)
$$

where again $\delta_{Q}(P)$, the delta function, is a $(1,1)$-form at $P$. (3.4) is easily shown by integrating on a small disk and using Stokes' theorem.

The classical stress tensor of (3.2) is

$$
T=-8 \pi^{2} \partial \varphi \partial \varphi
$$

a $(2,0)$-tensor. Its quantum version looks the same but with normal ordering to remove self-contractions. $T$ is defined so that in operator products [3]

$$
T(z) \psi(w) \sim \frac{h}{(z-w)^{2}} \psi+\text { less singular terms . }
$$

where $h$ is the spin of $\psi$. One can check the normalization of (3.5) by showing that $\partial \varphi$ has spin one. Then

$$
T(z) \mathrm{e}^{ \pm q \varphi(w)} \sim\left(\frac{1}{z-w}\right)^{2} \frac{q^{2}}{16 \pi^{2}} \cdot\left(-\frac{1}{2}\right)+\cdots .
$$

Thus choosing $q=4 \pi i$ gives $b, c$ of spin one half.

It is now simple to show the equivalence of (3.1) and (3.2), still in spin 1/2. First, the zero-point functions agree up to an overall multiplicative constant:

$$
Z_{b}[g]=\text { const. } \cdot Z_{f}[g]
$$

Each side of (3.6) is a functional of a metric chosen on $\Sigma$ to regularize the theories. (3.6) holds because both bosons and nonchiral fermions are free of gravitational anomalies, and 
both sides have the same anomalous variation under Weyl transformations (see sect. 5.A). Since on the sphere every metric $g$ is related to any reference $g_{0}$ by coordinate and Weyl transformations [16], we see that (3.6) really does hold up to a constant. As for the higher correlation functions, they follow from the fact that

$$
\begin{aligned}
\left\langle\mathrm{e}^{4 \pi i \varphi(z)} \mathrm{e}^{-4 \pi i \varphi(w)}\right\rangle & =\exp \left[16 \pi^{2}\langle\varphi(z) \varphi(w)\rangle\right] \sim \frac{1}{|z-w|^{2}} \\
& \sim\langle b \bar{b}(z) c \bar{c}(w)\rangle .
\end{aligned}
$$

Next we relax the condition of spin $\lambda=\frac{1}{2}$, to fields $b$ of spin $\lambda$ and $c$ of spin $1-\lambda$. Clearly (3.6) cannot hold as it stands, since we have not told $Z_{b}$ about $\lambda$. Some modification of (3.2) is needed in order to specify what spin we wish to bosonize. The correct choice is $S=S_{1}+S_{2}$, where

$$
S_{2}=(1-2 \lambda) \int R_{K}^{\varphi}
$$

The easiest way to check the normalization of (3.8) is to note that for $\lambda>1$, say, $Z_{f}$ is actually zero due to the presence of zero modes of $b$ and c. Using (2.18) with $g=0$ we see that $b$ has $1-2 \lambda$ more zero modes than $c$. Hence we get nonzero answers only if we consider correlation functions with $1-2 \lambda$ more insertions of $b$ than of $c$. To reproduce this behavior in the bosonic system note that the functional integral using (3.8) also vanishes for the zero point function: integrating over the constant mode $\varphi_{0}$ of $\varphi$ gives $\int_{0}^{2 \pi} \exp \left[(1-2 \lambda) \varphi_{0} \int R_{K}\right]$, which vanishes by (2.28). To get a nonzero answer we must insert $b$ and $\bar{b}$ fields, to get

$$
\int[\mathrm{d} \varphi] \mathrm{e}^{-\left(S_{1}+S_{2}\right)} \prod_{i=1}^{k} \mathrm{e}^{4 \pi i \varphi\left(P_{i}\right)}
$$

which with (3.8) is indeed zero unless $k=1-2 \lambda$.

(3.8) modifies the stress tensor by adding $-2 \pi i(2 \lambda-1) \partial^{2} \varphi$ to it [3]. Computing the operator product one again finds $q=4 \pi i$ in (3.3). With the modified action (3.6) continues to hold on the sphere for any spin, since (as we will check later) with $S_{2}$ both sides again have the same Weyl transformation and this again suffices on the sphere.

\section{B. The Torus}

When we move up in complexity from the sphere to the torus we at once encounter 
two novel features. First, it is no longer true that every metric is related to every other by coordinate and Weyl symmetries: a residual "moduli space" of inequivalent metrics remains [16]. Secondly, in any given degree there is now a wide variety of inequivalent bundles over $\Sigma$ in which $b$ and $c$ could take values. Thus we not only need to tell the bosons what spin they are to mimic; we must also tell them about a point in the jacobian $J(\Sigma)$ describing the twists of $b, c$. Fortunately on the torus we still have a canonical formalism, which we can use to address the problem.

On the torus every spin bundle has degree $2 \lambda(g-1)=0$, so we can take a fiat metric, $R_{K}=0$. Then $S_{2}=0$. A spin bundle $L$ is a flat bundle whose square is trivial; we can parametrize the four possible choices by measuring the difference between $L$ and one particular spin bundle, the trivial one. If we take the torus defined by the unit square in $C$, we then have

$$
\begin{aligned}
& b(1)=\mathrm{e}^{2 \pi i \theta} b(0) \\
& b(i)=\mathrm{e}^{-2 \pi i \phi} b(0)
\end{aligned}
$$

Here $\theta, \phi$ give the holonomy of the flat bundle $L$ as in (2.29) and (2.39). In this introductory section we will restrict to untwisted spin bundles, i.e. $\theta, \phi=0$ or $\frac{1}{2}$. The field $c$ then lives in the bundle $K \otimes L^{-1} \cong L$. Also we will not consider $\theta=\phi=0$ since with this choice $Z_{f}=0$ due to the zero mode; that is, we consider only the three "even" spin structures.

Certainly (3.6) cannot hold as it stands on the torus, since again one side depends on $\theta, \phi$ while the other does not. Instead one expects that the bosonic theory with action $S_{1}$ should give the sum over all spin structures of the corresponding fermionic theories. Detailed calculation affirms this expectation [6] (see also sect. 4.C). To bosonize just one spin structure one must add to $S_{1}$ a new term $S_{3}$ depending on $\theta, \phi$. We will find $S_{3}$ by canonically quantizing and applying the physical lesson that fermions correspond to solitons of the field $\varphi$.

Since moduli will not play an important role in this subsection we will again take the torus to be the unit square in $C$, with identifications and (3.10). We will quantize with euclidean time running up the imaginary axis. Then the partition functions $Z_{f}(\theta, \phi)$ are traces over the Ramond and Neveu-Schwarz Hilbert spaces, for $\theta=0, \frac{1}{2}$ respectively. $\phi$ 
on the other hand denotes the boundary conditions in time. We then have

$$
\begin{aligned}
& Z_{f}\left(\frac{1}{2}, \frac{1}{2}\right)=T r_{N S} \mathrm{e}^{-H} \\
& Z_{f}\left(\frac{1}{2}, 0\right)=T r_{N S}(-)^{F} \mathrm{e}^{-H}
\end{aligned}
$$

Here $F$ is the fermion number operator. Hence $Z_{f}\left(\frac{1}{2}, \frac{1}{2}\right) \pm Z_{f}\left(\frac{1}{2}, 0\right)$ is a trace over the even (resp. odd) fermion-number space. It must therefore in the bosonic language receive contributions only from states of even (resp. odd) soliton number.

Recall that the soliton number of a field configuration $\varphi$ is $2 n$, where the cohomology class of $\mathrm{d} \varphi$ is $n \alpha+m \beta$, so that a functional integral over $\varphi$ includes a sum over all soliton sectors. Thus our modification to the action $S_{3}$ must have the effect of weighting the various winding sectors in such a way as to cancel the odd-soliton contributions to $Z_{b}\left(\frac{1}{2}, 0\right)+Z_{b}\left(\frac{1}{2}, \frac{1}{2}\right)$, and so on. A possible set of weighting factors is shown in $F$ ig. 5 . In the left column we have shown the spin structures for the fermionic system. On the right the boxes represent the contributions to the bosonic path integral from the winding sectors with $(n, m)=\left(\frac{1}{2} \cdot\right.$ even,$\frac{1}{2} \cdot$ even $),\left(\frac{1}{2} \cdot\right.$ even, $\frac{1}{2} \cdot$ odd $)$, and so on. Each box on the right thus represents a sum $Z_{b, i}^{\text {partial }}, i=1, \ldots 4$ over an infinite subclass of field configurations. The effects of $S_{3}$ are in the phases $\sigma_{i j}$ : we have $Z_{b}\left(\frac{1}{2}, 0\right)=\sum_{i=1}^{4} \sigma_{3 i} Z_{b, i}^{\text {partial }}$, etc.

\section{FERMIONS}

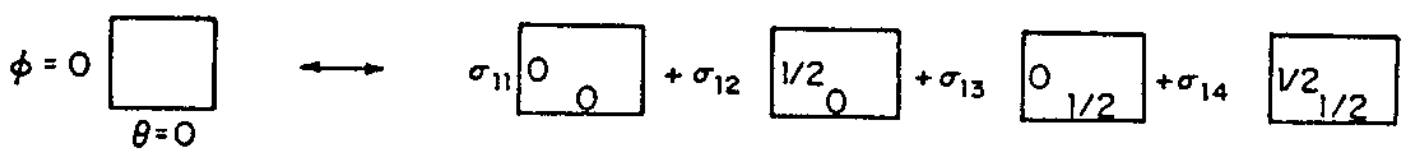

$\phi=1 / 2{ }_{\theta=0}$

$\phi=0 \prod_{\theta=1 / 2}$

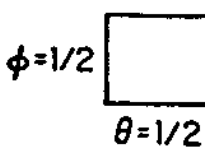

BOSONS
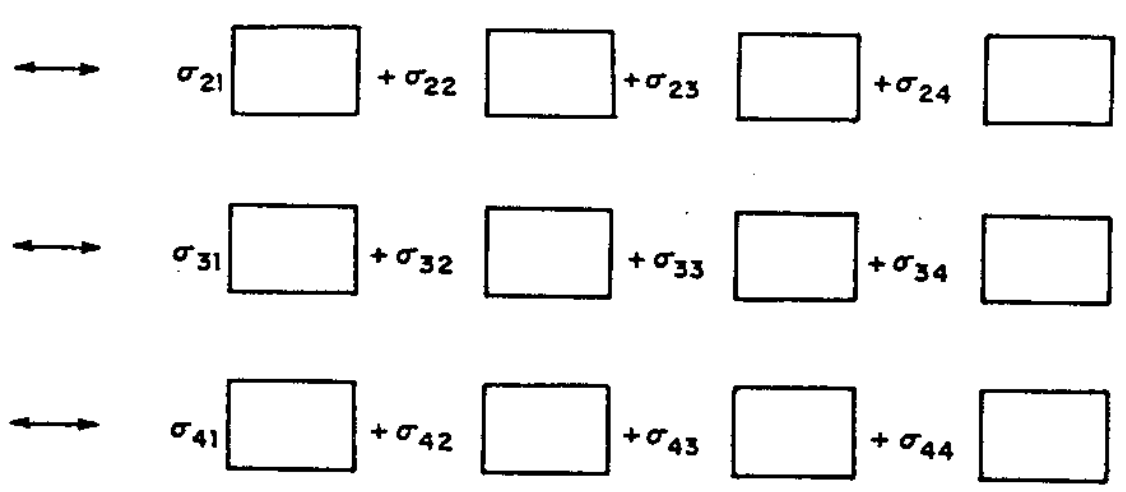

Fig. 5: Weighting the soliton sectors.

The conditions that $Z_{b}\left(\frac{1}{2}, 0\right) \pm Z_{b}\left(\frac{1}{2}, \frac{1}{2}\right)$ have no odd (resp. even) soliton contribution now imply that in these combinations $Z_{b, 3}^{\text {partial }}$ and $Z_{b, 4}^{\text {partial }}$ must cancel (resp. $Z_{b, 1}^{\text {partial }}$ 
and $\left.Z_{b, 2}^{\text {partial }}\right)$, i.e.

$$
\begin{array}{cc}
\sigma_{33}=-\sigma_{43}, & \sigma_{34}=-\sigma_{44} \\
\sigma_{31}=\sigma_{41}, & \sigma_{32}=\sigma_{42}
\end{array} .
$$

We can get further conditions by letting the torus degenerate with very long time. Then only the ground state contributes to the fermionic partition function. With zetafunction regularization the split ground state of the Ramond sector has nonzero energy, so that $Z_{f}(0, \phi) \rightarrow 0$. Take $\phi=\frac{1}{2}$. On the bosonic side, only the zero-soliton sectors contribute, but they do so independently of the time winding $2 m$, in the limit. Thus the two contributing partial sums $Z_{b, 1}^{\text {partial }}$ and $Z_{b, 2}^{\text {partial }}$ must cancel from $Z_{b}\left(0, \frac{1}{2}\right)$, so that $\sigma_{21}=-\sigma_{22}$.

We must also impose the condition of modular invariance on the $\sigma_{i j}$. Requiring for example that our prescription be unchanged when we exchange the roles of space and time gives relations like $\sigma_{21}=\sigma_{31}, \sigma_{22}=\sigma_{33}$, etc. Requiring that the torus with corners 0 , $1, i+2, i+1$ give the same answers as the unit square gives $\sigma_{31}=\sigma_{41}, \sigma_{33}=\sigma_{44}$, etc. These conditions fix $\sigma_{i j}, i \neq 1$ up to an overall constant, which we take to be unity: ${ }^{7}$

$$
\sigma_{i j}=\left\{\begin{array}{ll}
-1, & i=j \\
+1, & i \neq j
\end{array} .\right.
$$

We can restate (3.11) in a way which makes its modular invariance obvious. Note again that the four spin structures split into one "odd" one (the trivial bundle) and three "even" ones. The names indicate that the number of zero modes of $\bar{\partial}_{L}$ is odd $(=1)$ or even $(=0)$ in the respective cases [38]. Here $\bar{\partial}_{L}$ is the Cauchy-Riemann operator coupled to the holomorphic bundle $L[16]$. Note also that the 1 -form $\psi=\mathrm{d} \varphi$ corresponds to a flat bundle $F(\psi)$ as in Fig. 4. The prescription (3.11) simply says that we must add to $S_{1}$ the topological term

$$
S_{3}=i \pi \sigma(L \otimes F(\psi))
$$

where $\sigma\left(L^{\prime}\right)$ is 0 or 1 , depending on whether $L^{\prime}$ is even or odd. Note that $L \otimes F(\psi)$ really is a spin bundle, since $\psi$ is a half-integral class. Also note that the preferred spin bundle $L_{A}$ for a marking $A$ is always even [37].

\footnotetext{
7 We can use the same reasoning to fix the $\sigma_{1 j}$. However, to fix the relative sign of $\sigma_{1 j}$ relative to the others we must interpolate between the spin structures, as we do in the sequel.
} 
The proof that $S_{1}+S_{3}$ is the correct bosonic action, as well as the generalization to arbitrary twists $\theta, \phi$, will come after we generalize everything to genus $g \geq 1$. We emphasize, however that (3.2) and (3.12) are by now a very plausible prescription on the torus, and that in higher genus essentially no new physics will be needed.

\section{Higher Genus}

When $\Sigma$ has more than one handle we can no longer take a flat metric, so we can no longer ignore $S_{2}$ (3.8). Also, the trivial bundle no longer serves as a reference spin bundle, since now $g-1 \neq 0$. These two issues will give rise to interlocking subtleties which conspire to make the full action modular-invariant.

The full action should be invariant under constant shifts of $\varphi$ once we include in it terms for field insertions, as on the sphere (eqn (3.9)). We will write these terms as

$$
S_{4}=-4 \pi i\left[\sum_{i=1}^{p} \varphi\left(P_{i}\right)-\sum_{i=1}^{q} \varphi\left(Q_{i}\right)\right] \text {. }
$$

Extending the argument of section 3.A to any genus, counting fermionic zero modes shows that the numbers of insertions must satisfy

$$
p-q=(2 \lambda-1)(g-1)
$$

When (3.14) is satisfied, then the full action should depend only on the closed 1-form

$$
\psi=\mathrm{d} \varphi
$$

and not on $\varphi$ itself.

The only action term besides $S_{4}$ which is not shift-invariant is $S_{2}$. Thus we are again tempted to take $S_{2}$ to be as in (3.8), since again (2.18) gives shift-invariance when (3.14) holds. On a complicated surface like Fig. 1, however, (3.8) is problematic. Given $\psi$ we can recover $\varphi$ as a function on the cut surface $\Sigma_{c}$ by defining

$$
\varphi(P)=\int_{\hat{P}}^{P} \psi
$$

for any point $\hat{P} \in \Sigma$. Shift invariance says that it is immaterial which $\hat{P}$ we choose. However, if we deform slightly the curves used to cut $\Sigma$ into $\Sigma_{c}$, we run into problems. 


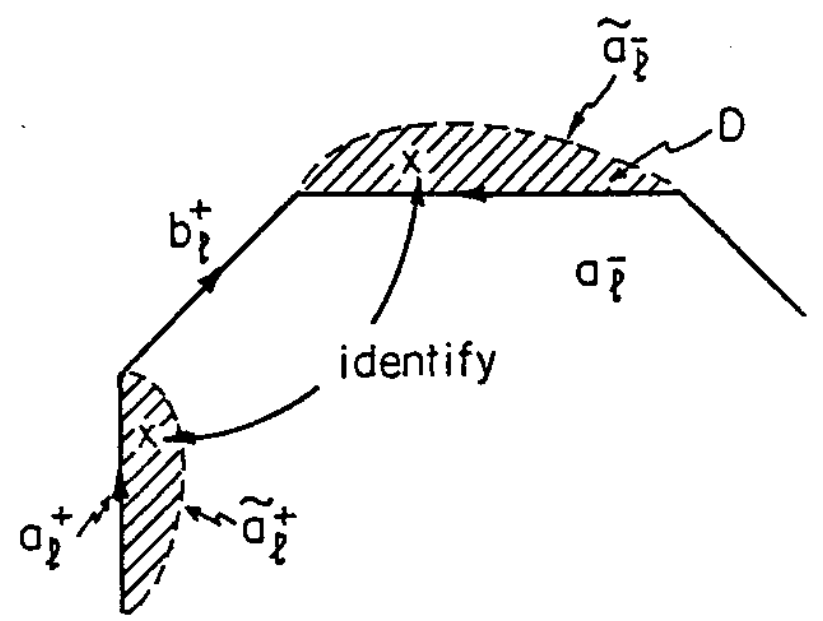

Fig. 6: Ambiguity of $S_{2}$ as we change the cut $a_{\ell}$ on $\Sigma$.

Since $\varphi$ in general jumps as we cross the cuts, we get an ambiguity in $S_{2}$ proportional to the integral of $R$ over the shaded region of Fig. 6 . Thus the action depends not only on the choice of a homology basis, but also on a choice of specific curves representing that basis.

We can repair the dependence on the curves by recalling (2.31). We need only to find an appropriate bundle $\xi$ and to add its holonomy around each cut, times the corresponding winding number of $\varphi$, to $S_{2}$. Specifically consider

$$
S_{2} \stackrel{?}{=}(1-2 \lambda) \int R_{K} \varphi+2 \pi i\left[m^{i} H\left(a_{i} ; \xi\right)-n^{i} H\left(b_{i} ; \xi\right)\right]
$$

As always $R_{K}$ is the curvature of the cotangent $K$ and we have expanded the real 1 -form $\psi=\mathrm{d} \varphi$ as $\psi=n \cdot \alpha+m \cdot \beta$. Then $S_{2}$ will be invariant when we displace the cuts if $\xi$ has spin $1-2 \lambda$. A natural choice for $\xi$ exists, namely $\mathcal{L}_{c} \otimes \mathcal{L}_{b}^{-1}$, where $\mathcal{L}_{b}$ is the line bundle where $b$ takes its values and $\mathcal{L}_{c}=K \otimes \mathcal{L}_{b}^{-1}$.

Unfortunately (3.16) is not well-defined, even modulo $2 \pi i$. The holonomy is defined $\bmod 1$, but $n^{i}, m^{i}$ are half-integers. Thus we would prefer to replace the holonomy of $\xi$ by $t$ wice the holonomy of some other bundle $\xi^{\prime}$ of spin $1 / 2-\lambda$. Since there is now no natural choice for $\xi^{\prime}$, we will let

$$
\mathcal{L} \equiv \mathcal{L}_{b}^{-1} \otimes L_{0}
$$

where $L_{0}$ is any even spin structure. Having introduced $L_{0}$ we will later have to show that the full action is independent of this choice. 
Our candidate for $S_{2}$ is thus

$$
S_{2}=2 \int R_{\mathcal{L}} \varphi-4 \pi i(\vec{n}, \vec{m}) \cdot J \cdot\left(\begin{array}{c}
H(\vec{a} ; \mathcal{L}) \\
H(\vec{b} ; \mathcal{L})
\end{array}\right)
$$

Eqn. (3.18) is invariant when we move the cuts in their homology classes, leaving fixed their common point, $P_{0}$. If we move $P_{0}$ itself, we can get a new system of cuts by choosing any curve $\gamma$ from $P_{0}$ to $\hat{P}_{0}$ and attaching it to each end of the existing cuts. Since the new $\hat{\Sigma}_{c}$ differs from the old by a set of measure zero, the first term of $S_{2}$ is unchanged; since each of the new curves traces and retraces $\gamma$, the other terms of (3.18) are also unchanged (See eq. (2.30)). Also (3.18) combined with (3.13) is still shift-invariant, i.e., a functional only of $\psi=\mathrm{d} \varphi$, since clearly this is separately true of the new terms of (3.18).

The only possible nonintrinsic information used in (3.18) is therefore the choice of $L_{0}$. We will return shortly to this dependence. First, however, we want to point out another important property of (3.18).

Often in instanton physics it is useful to divide the quantum field into a topologically nontrivial piece satisfying the equations of motion, plus a fluctuation piece which is topologically trivial. By requiring that the former piece stationarize the action we remove cross terms in the total action; for a quadratic system this splits $S_{1}$ cleanly into two terms:

$$
\begin{aligned}
\varphi & =\varphi_{n m}+\tilde{\varphi} \\
S_{1}[\varphi] & =S_{1}\left[\varphi_{n m}\right]+S_{1}[\tilde{\varphi}]
\end{aligned}
$$

where $\bar{\partial} \partial \varphi_{n m}=0$ and $\tilde{\varphi}$ is single-valued. For our case everything is especially simple, since (3.19) is the Hodge decomposition, and so the harmonic $\varphi_{n m}$ has no continuous collective coordinates. Indeed we have simply

$$
\psi_{n m} \equiv \mathrm{d} \varphi_{n m}=(n, m)\left(\begin{array}{l}
\alpha \\
\beta
\end{array}\right)
$$

Of course the linear term $S_{2}$ also splits similarly to (3.19).

In the remainder of this section we will focus on $S\left[\varphi_{n m}\right]$, leaving $S[\tilde{\varphi}]$ for later.

We can now evaluate $S_{2}\left[\varphi_{n m}\right]$ using any convenient metric. Let $D=\sum \ell_{i} T_{i}$ be any divisor representing $\mathcal{L}$ and $\sigma=1_{O(D)}$ the corresponding unit section. Then away from the poles and zeros of $\sigma$ the curvature of $\mathcal{L}$ is (2.27). Let $\Delta_{i}$ be small neighborhoods 
of the points $T_{i}$ and $\Sigma_{c}^{\prime}=\Sigma_{c}-U_{i} \Delta_{i}$. Then integrating $S_{2}$ by parts one has for small $\Delta_{i}$

$$
S_{2}=-2 \sum_{i} \oint_{\partial \Delta i} \varphi_{n m} \partial \log \|\sigma\|^{2}-2 \int_{\Sigma} \bar{\partial} \varphi_{n m} \wedge \partial \log \|\sigma\|^{2}
$$

The boundary terms from the cuts of $\Sigma_{c}$ have cancelled the explicit holonomy terms of (3.18). Integrating the second term again by parts gives zero, since $\varphi_{n m}$ is harmonic, $\bar{\partial} \varphi_{n m}$ is single-valued, and the boundary terms near $T_{i}$ go to zero. Near a zero of $\sigma$ we have $\partial \log \|\sigma\|^{2} \sim z^{-1} \mathrm{~d} z$, and so adding (3.13) we get

$$
\begin{aligned}
S_{2}+S_{4} & =-4 \pi i\left[\sum_{i} \ell_{i} \varphi_{n m}\left(T_{i}\right)+\sum_{i} \varphi_{n m}\left(P_{i}\right)-\sum_{i} \varphi_{n m}\left(Q_{i}\right)\right] \\
& =-4 \pi i\left[\sum_{i} \ell_{i} \int^{T_{i}} \psi_{n m}+\sum_{i}^{p} \int^{P_{i}} \psi_{n m}-\sum_{i}^{q} \int^{Q_{i}} \psi_{n m}\right] .
\end{aligned}
$$

The common starting point of these integrals does not matter since we have (3.14). Nor do the paths matter: changing a contour by a homologically trivial circuit gives zero since $\psi$ is closed, while changing it by a generator of $H_{1}(\Sigma)$ only changes $S_{2}+S_{4}$ by a multiple of $2 \pi i . S_{2}+S_{4}$ is also manifestly independent of any choice of homology basis. It does however still depend on the chosen even spin bundle $L_{0}$ via the divisor class of $D$. It also potentially depends on the divisor $D$ itself, not just on its class.

To show that $S_{2}+S_{4}$ depends only on the class of $D$, i.e., on the bundle $\mathcal{L}$, we will cast it in terms of the Jacobi map $I_{A}$ and use Abel's theorem. Note that any harmonic $\psi=(n, m)\left(\begin{array}{l}\alpha \\ \beta\end{array}\right)$ can be expressed in terms of the abelian differentials $\vec{\omega}_{\mathcal{A}}$ as

$$
\psi=\left(m-\bar{\tau}_{A} n\right)^{t} \cdot Y_{A} \cdot \vec{\omega}_{A}+c . c .
$$

(see (2.21)). To verify (3.21), integrate both sides around $a_{i}, b_{i}$. Inserting into (3.20) and using (2.26) we get

$$
S_{2}+S_{4}=-4 \pi i\left\{\left(m-\bar{\tau}_{A} n\right)^{t} \cdot Y_{A} \cdot I_{A}\left[D+D_{i n s}\right]+c . c .\right\}
$$

where

$$
D_{\text {ins }}=\sum_{i=1}^{p} P_{i}-\sum_{i=1}^{q} Q_{i}
$$


is the divisor built from the field insertion points. Thus $S_{2}+S_{4}$ depends only on the divisor class of $D+D_{\text {ins }}$. It is now straightforward to show that

$$
S_{2}+S_{4}=4 \pi i(n \cdot \phi+m \cdot \theta)
$$

where $I_{A}\left[D+D_{\text {ins }}\right] \equiv-\left(\phi+\tau_{A} \cdot \theta\right)$. One can derive (3.24) directly or using (2.33), (2.34).

We still have not generalized the term $S_{3}$ needed on the torus to distinguish the various spin structures. However, the parity $\sigma(L)$ of a spin bundle makes invariant sense in any genus. Thus we can generalize (3.12) to

$$
S_{3}=i \pi \sigma\left(L_{0} \otimes F(\psi)\right)
$$

(3.25) is really the only sensible generalization of (3.12). We can't, for example, use $\mathcal{L}$ in place of $L_{0}$, since $\sigma(L)$ is only defined for untwisted spin-1/2 bundles [37]. For the case considered in section 3.B, however, $\mathcal{L}_{b}$ is a bundle of this type. Then we can recover (3.12) from (3.25), (3.22) by taking $g=1, \lambda=1 / 2, D_{\text {ins }}=0$, and choosing $L_{0}=\mathcal{L}_{b}$ so that (3.22) is zero. ( $\mathcal{L}_{b}$ was called $L$ in (3.12).)

We have now completely defined the bosonic action $S_{b}=S_{1}+S_{2}+S_{3}+S_{4}$. One can readily show that this prescription is equivalent to the one given in [5].

\section{Consistency and Uniqueness}

We have arrived at a modular-invariant action in any genus which reduces to the kinetic term plus (3.8) on the sphere or (3.12) on the torus. The only potential problem with $S_{6}=S_{1}+S_{2}+S_{3}+S_{4}$ as a classical action is its dependence on an arbitrary even spin bundle $L_{0}$. To see that this dependence is trivial, we express $L_{0}$ as $L_{0}=L_{A} \otimes F(\hat{\psi})$ where $L_{A}$ is the preferred spin bundle for some homology basis $A$. In this basis we expand $\psi=\mathrm{d} \varphi$ as $\psi=(n, m)\left(\begin{array}{l}\alpha \\ \beta\end{array}\right)$ and $\hat{\psi}=(\hat{n}, \hat{m})\left(\begin{array}{l}\alpha \\ \beta\end{array}\right)$. Then the parity equals [37]

$$
\begin{aligned}
\sigma\left(L_{0} \otimes F(\psi)\right) & =\sigma\left(L_{A} \otimes F(\psi+\hat{\psi})\right)=4(n+\hat{n}) \cdot(m+\hat{m}) \quad(\bmod 2) \\
& =\sigma\left(L_{A} \otimes F(\psi)\right)+4(n \cdot \hat{m}+m \cdot \hat{n}) \quad(\bmod 2)
\end{aligned}
$$

since $L_{0}$ is itself even. At the same time, however, (3.24) changes. Using (2.32) the change in $\left(\begin{array}{l}\phi \\ \theta\end{array}\right)$ describing $\mathcal{L}$ is $-J\left(\begin{array}{l}\hat{n} \\ \hat{m}\end{array}\right)$, so that the change in $S_{2}+S_{3}$ equals

$$
4 \pi i(-n \cdot \hat{m}+m \cdot \hat{n})+4 \pi i(n \cdot \hat{m}+m \cdot \hat{n})=0 \quad(\bmod 2 \pi i)
$$


Thus the action $S_{b}$ is completely intrinsic.

How unique is the bosonic action? Any modification to $S_{b}$ must have only topological terms, since $S_{b}$ is locally correct. It must also be intrinsically defined, i.e., modular invariant. Also, to preserve clustering any new global terms in $S$ must factorize as a complicated Riemann surface pinches off into many tori. One can see from (3.24), (3.26) that our candidate action already has this property. If we weight each winding sector by an additional phase $E(\vec{n}, \vec{m})$, the requirement of factorization says that

$$
E(\vec{n}, \vec{m})=\prod_{i=1}^{g} \epsilon\left(n^{i}, m^{i}\right)
$$

for some universal function $\epsilon$. The analysis needed to fix $\epsilon$ has been done in [48]; we will now summarize the relevant case.

We can constrain $\epsilon(n, m)$ by requiring $E$ to be invariant under the modular transformation given by

$$
\Lambda=\left[\begin{array}{cccc}
1 & 0 & 0 & 0 \\
0 & 1 & 0 & 0 \\
-1 & 1 & 1 & 0 \\
1 & -1 & 0 & 1
\end{array}\right]
$$

Using (3.27) in genus 2 this says that

$$
\epsilon\left(n_{1}+m_{1}-m_{2}, m_{1}\right) \epsilon\left(n_{2}-m_{1}+m_{2}, m_{2}\right)=\epsilon\left(n_{1}, m_{1}\right) \epsilon\left(n_{2}, m_{2}\right)
$$

Without loss of generality we can let $\epsilon(0,0)=1$. Also, one-loop modular invariance requires

$$
\epsilon(d n+c m, b n+a m)=\epsilon(n, m), \quad a d-b c=1 .
$$

Taking $\left(\begin{array}{ll}a & b \\ c & d\end{array}\right)=\left(\begin{array}{cc}1 & -1 \\ 0 & 1\end{array}\right)$ and $\left(\begin{array}{ll}1 & 0 \\ 1 & 1\end{array}\right)$ we find

$$
\begin{array}{r}
\epsilon(n, m-n)=\epsilon(n, m) \\
\epsilon(n+m, m)=\epsilon(n, m)
\end{array}
$$

so

$$
\epsilon\left(n_{1}-m_{2}, m_{1}\right) \epsilon\left(n_{2}-m_{1}, m_{2}\right)=\epsilon\left(n_{1}, m_{1}\right) \epsilon\left(n_{2}, m_{2}\right)
$$

Taking $n_{1}=m_{1}=n_{2}=0$ in (3.28) gives $\epsilon\left(-m_{2}, 0\right)=1$. Thus

$$
\epsilon(n, n)=\epsilon(n, 0)=\epsilon(0, n)=\epsilon(0,0)=1
$$


Taking $n_{1}=n_{2}=0$ in (3.28) gives

$$
\epsilon(n, m)=\epsilon(-m,-n)^{-1}
$$

Setting $n_{2}=0$,

$$
\epsilon\left(n+n^{\prime}, m\right)=\epsilon(n, m) \epsilon\left(n^{\prime}, m\right)
$$

and similarly $\epsilon\left(n, m+m^{\prime}\right)$. Thus $\epsilon(n, m)=\epsilon(1,1)^{n m}$. But by $(3.29), \epsilon(1,1)^{n^{2}}=1$ for every $n$. Thus $\epsilon(1,1)=1$, and the action is unique.

\section{Bosonization Formulæe}

\section{A. Recap}

The bosonization of the first-order fermionic system thus proceeds as follows. If $b, c$ are fields of spin $\lambda, 1-\lambda$ with action

$$
S_{f}=\frac{i}{2 \pi} \int_{\Sigma}(b \bar{\partial} c+\bar{b} \partial \bar{c})
$$

then we introduce a Bose theory with field $\varphi$ well-defined up to half-integers and action ${ }^{8}$

$$
\begin{gathered}
S_{b}=S_{1}+S_{2}+S_{3} \\
S_{1}=4 \pi i \int_{\Sigma} \partial \varphi \wedge \bar{\partial} \varphi \\
S_{2}=2 \int R_{\mathcal{L}} \varphi+4 \pi i \sum_{i}\left[m^{i} H\left(a_{i} ; \mathcal{L}\right)-n^{i} H\left(b_{i} ; \mathcal{L}\right)\right] \\
S_{3}=i \pi \sigma\left(L_{0} \otimes F(\mathrm{~d} \varphi)\right) .
\end{gathered}
$$

Here $\mathcal{L}$ is the line bundle

$$
\mathcal{L} \equiv \mathcal{L}_{b}^{-1} \otimes L_{0}
$$

$\mathcal{L}_{b}$ is the bundle of degree $2 \lambda(g-1)$ where $b$ takes values, and $L_{0}$ is any even spin bundle. $R_{\mathcal{L}}$ and $H(\cdot ; \mathcal{L})$ are the curvature and holonomy of $\mathcal{L}$, and $n_{i}, m_{i}$ are the winding numbers of the field configuration $\varphi$ about the cycles $a_{i}, b_{i}, F(\mathrm{~d} \varphi)$ is the flat

8 O. Alvarez has told us that the terms $S_{2}$ and $S_{3}$ can also be understood in terms of corrections to the heat kernel on the cut Riemann surface. 
line bundle with holonomy given by the one-form $\mathrm{d} \varphi$, and $\sigma(L)$ is the parity of a spin bundle $L . S_{b}$ is well-defined modulo $2 \pi i$ once we include appropriate insertions of fields.

The bosonization results we seek to establish say that these two systems have the same correlation functions up to an overall multiplicative constant under the correspondence

$$
b(P) \bar{b}(P) \propto \mathrm{e}^{4 \pi i \varphi(P)}, \quad c(Q) \bar{c}(Q) \propto \mathrm{e}^{-4 \pi i \varphi(Q)}
$$

We need $p$ insertions of the first kind and $q$ of the second kind, where

$$
p-q=(2 \lambda-1)(g-1)
$$

Sometimes we consider the insertions as a term $S_{4}$ in the action (eqn. (3.13)).

(3.3) is not yet completely specified. Here we have some latitude, since neither have we yet specified the normal-ordering prescription to be used in evaluating bosonic correlators. The simplest prescription to use is a coordinate-invariant one, in which all coincident Green functions are replaced by

$$
G_{r}(P, Q) \equiv \lim _{P \rightarrow Q}[G(P, Q) / d(P, Q)]
$$

$d(P, Q)$ is the metric distance between two points of $\Sigma . G(P, Q)$ is the Green function defined using the given metric in (2.44)-(2.46).

One reason why (4.1) is so nice is that for the Arakelov metric we have

$$
\log G_{r}(P, P)=0
$$

(4.2) follows since the Arakelov metric $\|\cdot\|_{\text {Arak }}$ is by definition the one for which the metric $\|\cdot\|^{\prime}$ defined by (2.51) again reproduces $\|\cdot\|_{\text {Arak }}$. However we will see that (4.1) is the correct prescription for any metric.

With (4.1) all bosonic correlations will be coordinate scalars. Since $b \bar{b}$ is not a scalar but a $(\lambda, \lambda)$-form, the precise statement of $(3.3)$ is that $e^{4 \pi i \varphi(P)}$ should correspond to ${ }^{9}$

$$
\|b(P)\|^{2} \equiv\left[g^{z \bar{z}}(P)\right]^{\lambda} b(P) \bar{b}(P), \quad\|c(Q)\|^{2} \equiv\left[g^{z \bar{z}}(Q)\right]^{1-\lambda} c(Q) \bar{c}(Q)
$$

or

$$
\left\langle\left\|b\left(P_{1}\right)\right\|^{2} \cdots\left\|b\left(P_{p}\right)\right\|^{2}\left\|c\left(Q_{1}\right)\right\|^{2} \cdots\left\|c\left(Q_{q}\right)\right\|^{2}\right\rangle_{f} \stackrel{?}{=}\left\langle\mathrm{e}^{4 \pi i \varphi\left(P_{1}\right)} \ldots \mathrm{e}^{-4 \pi i \varphi\left(Q_{q}\right)}\right\rangle_{b} \quad .
$$

9 We will later comment on alternatives to (4.1). 
In the succeeding subsections we write out both sides of (4.4) in some detail to get a set of identities expressing the mathematical content of bosonization.

Note that (4.4) is a nonchiral amplitude. Once we have proven it we can modify (4.1) to eliminate the metric factors in (4.4), then take the holomorphic square root in the variables $P_{i}, Q_{i}$ on both sides of the formula. In a sense this gives a bosonic formula for the chiral amplitude on the left hand side. This is not however the same thing as presenting a bosonic theory which, without any modifications, reproduces chiral amplitudes. We do not know how to write a bosonic theory whose correlation functions have the appropriate geometrical meaning to do this.

\section{B. Fermion Correlations}

We begin with the left side of (4.4). If there are no insertions (this can happen only if the spin $\lambda=\frac{1}{2}$ ), then the rules of functional Grassmann integration say that the zero-point function is just the functional determinant of $\bar{\partial}_{\mathcal{L}}^{\dagger} \bar{\partial}_{\mathcal{L}}$. We will always use zetafunction regulation for determinants, as it fits in best with the methods of sec. 5 . If there are only insertions of $b$ then the p-point function is the antisymmetrized product of the $p$ zero modes of $\bar{\partial}_{\mathcal{L}}$. For example, if the spin $\lambda$ and the genus $g$ are both greater than 1 , then the degree of $\mathcal{L}_{c}$ is negative and so $\bar{\partial}_{\mathcal{L}_{0}}$ has no zero modes. Then we can have $q=0$ insertions of $c$ and so, by (2.18), $p=(2 \lambda-1)(g-1)$ insertions of $b$ :

$$
\left\langle b\left(P_{1}\right) \bar{b}\left(P_{1}\right) \cdots b\left(P_{p}\right) \bar{b}\left(P_{p}\right)\right\rangle_{f}=\frac{\operatorname{det}^{\prime} \bar{\partial}_{\mathcal{L}_{b}}^{\dagger} \bar{\partial}_{\mathcal{L}_{b}}}{\operatorname{det}\left(u_{i}, u_{j}\right)} \cdot\left|\sum_{\pi}(-)^{\pi} u_{1}\left(P_{\pi(1)}\right) \cdots u_{p}\left(P_{\pi(p)}\right)\right|^{2} \text {. }
$$

Here $u_{1}, \cdots u_{p}$ are the $p$ zero modes of $\bar{\partial}_{\mathcal{L}_{b}}$ and $(\cdot, \cdot)$ is the inner product on sections of $\mathcal{L}_{b} . \pi$ runs over permutations, and so the factor inside the absolute square lives in $\wedge^{\max }\left(\left.\oplus_{i=1}^{p} \mathcal{L}_{b}\right|_{P_{i}}\right)$. We will denote this factor by $\operatorname{det} u_{i}\left(P_{j}\right)$. Using the given norm on $\mathcal{L}_{b}$ we can now write the left side of (4.4) as

$$
\left\langle\left\|b\left(P_{1}\right)\right\|^{2} \cdots\left\|b\left(P_{p}\right)\right\|^{2}\right\rangle_{f}=\frac{\operatorname{det}^{\prime} \bar{\partial}_{\mathcal{L}_{b}}^{\dagger} \bar{\partial}_{\mathcal{L}_{b}}}{\operatorname{det}\left(u_{i}, u_{j}\right)} \cdot\left\|\operatorname{det} u_{i}\left(P_{j}\right)\right\|^{2} .
$$

If $\lambda=1$ then $\bar{\partial}_{\mathcal{L}_{c}}$ still has no zero modes unless $\mathcal{L}_{b}$ is untwisted, i.e. $\mathcal{L}_{b}=K$. In this case $\mathcal{L}_{c}$ is trivial and so has the constant zero mode $v_{0} \equiv 1$. Its norm is $(1,1)=A_{\Sigma}$, the area of $\Sigma$ in the given metric, while the factor $\left\|v_{0}(Q)\right\|^{2}=1$. We thus get

$$
\left\langle\left\|b\left(P_{1}\right)\right\|^{2} \cdots\left\|b\left(P_{p}\right)\right\|^{2}\|c(Q)\|^{2}\right\rangle_{f}=\frac{\operatorname{det}^{\prime} \bar{\partial}^{\dagger} \bar{\partial}}{\operatorname{det}(i Y)^{-1} \cdot A_{\Sigma}} \cdot\left\|\operatorname{det} \omega^{i}\left(P_{j}\right)\right\|^{2} .
$$


Here we have used the fact that for spin one the $u_{i}$ are the abelian differentials $\omega^{i}$ and $\left(\omega^{i}, \omega^{j}\right)=i \int \bar{\omega}^{i} \wedge \omega^{j}=i \oint_{a_{k}} \bar{\omega}^{i} \oint_{b_{k}} \omega^{j}-(a \leftrightarrow b)=i\left(\tau_{i j}-\bar{\tau}_{i j}\right) \equiv i\left(Y^{-1}\right)_{i j}$. Also $\operatorname{det}^{\prime} \bar{\partial}_{K}^{\dagger} \bar{\partial}_{K}=\operatorname{det}^{\prime} \bar{\partial}^{\dagger} \bar{\partial}$ since the nonzero eigenvalues of these operators are the same.

For $\lambda<\frac{1}{2}$ we interchange the roles of $b$ and $c$.

Finally we can consider the case when more than the minimal number of inserted fields is present. For this we need the fermionic Green function, which is in general more complicated than the spin-1/2 version (see (3.7), [49]) due to the presence of zero modes. Suppose again that $\bar{\partial}_{\mathcal{L}_{c}}$ has no zero modes, i.e. $H^{0}\left(\Sigma ; \mathcal{L}_{c}\right)=0$. Let $P_{1}$ be the projector to the space orthogonal to the zero modes of $\bar{\partial}_{\mathcal{L}_{b}}$. We can unambiguously invert $\bar{\partial}_{\mathcal{L}_{b}}$ restricted to this space; call the inverse $\mathcal{G}$. Thus

$$
\begin{aligned}
& \mathcal{G} \circ \bar{\partial}_{\mathcal{L}_{b}}=\mathbf{P}_{1} \\
& \bar{\partial}_{\mathcal{L}_{b}} \circ \mathcal{G}=1
\end{aligned}
$$

Mathematically $\mathcal{G}$ is the "parametrix" of $\bar{\partial}_{\mathcal{L}_{b}}$, an inverse up to a finite-rank term. Its existence is guaranteed by Hodge theory[39]. We can represent $\mathcal{G}$ by an integral kernel $\mathcal{G}(P, Q)$. For fixed $Q, \mathcal{G}(\cdot, Q)$ is a section of $\mathcal{L}_{b} \otimes \mathcal{O}(Q) \otimes \mathcal{L} \mathcal{d}_{Q}$. Its residue at $Q=P$ is therefore a pure number by (2.52), namely $1 / 2 \pi i$.

$\mathcal{G}$ is the basic $b c$ contraction. With it we get

$$
\begin{aligned}
& \left\langle\left\|b\left(P_{1}\right)\right\|^{2} \cdots\left\|b\left(P_{p}\right)\right\|^{2}\left\|c\left(Q_{1}\right)\right\|^{2} \cdots\left\|c\left(Q_{q}\right)\right\|^{2}\right\rangle_{f}= \\
& =\frac{\operatorname{det}^{\prime} \bar{\partial}_{\mathcal{L}_{b}}^{\dagger} \bar{\partial}_{\mathcal{L}_{b}}}{\operatorname{det}\left(u_{i}, u_{j}\right)} \cdot\left\|\operatorname{det}\left(\begin{array}{cccccc}
u_{1}\left(P_{1}\right) & \cdots & u_{p-q}\left(P_{1}\right) & \mathcal{G}\left(P_{1}, Q_{1}\right) & \cdots & \mathcal{G}\left(P_{1}, Q_{q}\right) \\
\vdots & & \vdots & \vdots & & \vdots \\
u_{1}\left(P_{p}\right) & \cdots & u_{p-q}\left(P_{p}\right) & \mathcal{G}\left(P_{p}, Q_{1}\right) & \cdots & \mathcal{G}\left(P_{p}, Q_{q}\right)
\end{array}\right)\right\|^{2}
\end{aligned}
$$

The large matrix is square, and its determinant is a vector in

$$
\left[\left.\bigotimes_{i=1}^{p} \mathcal{L}_{b}\right|_{P_{i}}\right] \otimes\left[\left.\bigotimes_{j=1}^{q} \mathcal{L}_{c}\right|_{Q_{j}}\right] \text {. }
$$

In essence what has happened is that we have manufactured $q$ additional zero modes of $\bar{\partial}_{\mathcal{L}_{b}}$. These extra modes $\mathcal{G}\left(\cdot, Q_{i}\right)$ have poles, but this is permitted since unlike $(4.5)$, the left side of (4.8) is supposed to have poles.

Similarly one can generalize (4.6) to the analog of (4.8) when $\lambda=1$. For this we must replace the unit operator in (4.7) by the projector $P_{2}$ to the complement of the zero mode space of $\bar{\partial}_{K}^{\dagger}$. 


\section{Instanton Sums}

We mentioned earlier that the bosonic amplitude splits into the product of a topological part times a fluctuation part when we split $\varphi$ as in (3.19). In this subsection we work out the former piece.

Recall that in section 3.B we argued that an extra topological term in the action was necessary to bosonize a single spin structure. Since the fermionic amplitudes for the different spin structures differ by theta-function factors (e.g. [6]), we want the topological part of the bosonic amplitude to be the absolute square of a theta function for one single characteristic, not the sum over spin structures obtained when the topological terms are omitted from the action [6]. The key result of this subsection is that indeed this is what happens.

It will be convenient for explicit computations to choose a homology basis $A$. We have seen that the action is independent of the choice of $A$. Since $A$ will not change, we will sometimes drop it from the notation.

First we substitute (3.21) into (3.2):

$$
S_{1}\left[\varphi_{n m}\right]=4 \pi i(m-\bar{\tau} n)^{t} \cdot Y \cdot(m-\tau n)
$$

Next we will make a specific choice for the arbitrary even spin bundle in (3.17), namely $L_{0}=L_{A}$, the preferred spin bundle for the homology basis $A$. We have already worked out $S_{2}+S_{4}$ for harmonic $\varphi_{n m}$ (eqn. (3.22)):

$$
S_{2}+S_{4}=4 \pi i\left[(m-\bar{\tau} n)^{t} \cdot Y \cdot z+c . c .\right]
$$

where (eqn. (3.17))

$$
z \equiv I\left[\mathcal{L}_{b} \otimes L_{A}^{-1} \otimes O\left(-D_{\text {ins }}\right)\right]
$$

Again $D_{i n s}$ is the divisor of insertion points (3.23) and we have written a bundle instead of its divisor class. Parenthetically we note that the topological part of the action can be simply expressed in terms of the natural hermitian form $B$ in (2.33): for harmonic $\varphi$ we have

$$
S_{1}+S_{2}+S_{4}=2 \pi[B(F(\mathrm{~d} \varphi), F(\mathrm{~d} \varphi))+B(F(\mathrm{~d} \varphi), z)-B(z, F(\mathrm{~d} \varphi))]
$$


We will not make explicit use of this form of the action.

Taking $L_{0}$ to be $L_{A}$ has the advantage of making $S_{3}$ very simple. By (3.26)

$$
S_{3}=4 \pi i n \cdot m
$$

Note that $\mathrm{e}^{-S_{3}}$ depends only on the values of $n$ and $m$ modulo 1 .

We wish to compute

$$
Z_{\text {inst }} \equiv \sum_{\mathrm{d} \varphi_{n m} \in H^{1}\left(\Sigma ; \frac{1}{2} \mathrm{Z}\right)} \mathrm{e}^{-S\left[\varphi_{n m}\right]}
$$

and in particular to show that it is the absolute square of a theta function. ${ }^{10}$ We know however that $\vartheta$ is defined by a sum over integer, not half-integer, vectors. Accordingly we will define the $2^{2 g}$ partial sums

$$
Z_{\nu, \mu} \equiv \sum_{k, \ell \in Z^{g}} \mathrm{e}^{-\left(S_{1}+S_{2}+S_{4}\right)[n=k+\nu, m=\ell+\mu]}
$$

where every entry of $\vec{\nu}, \vec{\mu}$ equals 0 or $\frac{1}{2}$. We omitted $\mathrm{e}^{-S_{3}}$ from (4.10) because as noted it is a function only of $\nu, \mu$. Thus

$$
Z_{\text {inst }}=\sum_{\nu, \mu \in\left(\mathrm{Z}_{2}\right)^{g}} \mathrm{e}^{4 \pi i \nu \cdot \mu} Z_{\nu, \mu}
$$

$Z_{2}$ denotes the group with two elements: $\left(\frac{1}{2} Z\right) / Z$.

In the remainder of this subsection we will prove the following formula for $Z_{\nu, \mu}$ :

$$
Z_{\nu, \mu}=2^{-3 g / 2}(\operatorname{det} 2 i Y)^{-\frac{1}{2}} \cdot \mathrm{e}^{-4 \pi i y Y y} \sum_{\epsilon, \zeta \in\left(Z_{2}\right)^{\diamond}} \mathrm{e}^{4 \pi i \epsilon \cdot \zeta} \vartheta\left[\begin{array}{c}
\nu+\epsilon \\
-\mu+\varsigma
\end{array}\right](-z \mid \tau) \overline{\vartheta\left[\begin{array}{c}
\nu+\epsilon \\
-\mu+\varsigma
\end{array}\right](z \mid \tau)}
$$

where $z=x+i y$ defines $y$. It is not hard to generalize (4.12) to a form useful for nonabelian bosonization and toroidal compactification.

Before proving (4.12), let us pause to see why it is just what we want. Substituting in (4.11), we get

$$
Z_{\text {inst }}=(\text { prefactor }) \cdot \sum_{\nu, \mu, \alpha, \beta} \mathrm{e}^{4 \pi i(\nu \cdot \mu+(\alpha-\nu)(\beta+\mu))} \vartheta\left[\begin{array}{l}
\alpha \\
\beta
\end{array}\right](-z \mid \tau) \overline{\vartheta\left[\begin{array}{l}
\alpha \\
\beta
\end{array}\right](z \mid \tau)} .
$$

10 The derivation below extends easily from the case of a single fermion (the lattice of integers) to many fermions (an arbitrary self-dual lattice). 
We have changed variables from $\epsilon, \zeta$ to $\alpha=\nu+\epsilon, \beta=-\mu+\zeta$. Then

$$
\begin{aligned}
Z_{\text {inst }} & =\text { (prefactor) } \cdot \sum_{\alpha, \beta} \vartheta \bar{\vartheta} \cdot \mathrm{e}^{4 \pi i \alpha \cdot \beta} \sum_{\mu} \mathrm{e}^{4 \pi i \alpha \cdot \mu} \cdot \sum_{\nu} \mathrm{e}^{-4 \pi i \beta \cdot \nu} \\
& =(\operatorname{det} i Y)^{-\frac{1}{2}} \mathrm{e}^{-4 \pi i y Y y}|\vartheta(z \mid \tau)|^{2}
\end{aligned}
$$

as desired. In terms of $\mathcal{N}$ in (2.43), this is ${ }^{11}$

$$
Z_{\text {inst }}=(\operatorname{det} i Y)^{-\frac{1}{2}} \mathcal{N}(z)
$$

Using (2.41) and (2.24) we see that $Z_{\text {inst }}$ is independent of the chosen marking, as we have already noted on general grounds.

We can also recover from (4.12) the answer one gets by omitting the topological terms from the action. Setting $z=0$ and dropping the weighting factor from (4.11), one indeed finds that $Z_{\text {inst }}$ is then proportional to the sum of the squares of all the even theta functions [6].

The general strategy for proving (4.12) is to diagonalize the action into a sum over two integer vectors $\vec{a}, \vec{b}$ of a function of $\vec{a}$ times a function of $\vec{b}$. Roughly speaking we will accomplish this diagonalization by "rotating $\vec{k}, \vec{\ell}$ by $45^{\circ}$." Then $Z_{\nu, \mu}$ becomes essentially the product of two factors, each of which turns out to be a theta function. The tricky part of the procedure lies in the idea of "rotating" a square lattice; this is where $\epsilon, \zeta$ will enter.

We start with the observation that

$$
\sum_{j \in Z} \mathrm{e}^{-2 \pi i j t}=\sum_{\ell \in Z} \delta(t-\ell)
$$

Regarding both sides as functions on $S^{1}$ this is just the Fourier transform of the deltafunction at the origin. Thus for a nice function $f$,

$$
\sum_{\ell \in Z^{*}} f(\ell+\mu)=\sum_{j \in Z^{\theta}} \mathrm{e}^{2 \pi i j \cdot \mu} \int \mathrm{d}^{g} t \mathrm{e}^{-2 \pi i j \cdot t} f(t),
$$

the "Poisson resummation formula." We will apply this to the sum over $\ell$ in (4.10).

Let $\tau=\tau_{1}+i \tau_{2}, z=x+i y$, and $S_{1}+S_{2}+S_{4}=2 \pi\left(S^{\prime}+S^{\prime \prime}\right)$, where

$$
S^{\prime}(m, n)=m \tau_{2}^{-1} m-2 n \tau_{1} \tau_{2}^{-1} m+2 i m \tau_{2}^{-1} y
$$

$11 Z_{\text {inst }}$ is essentially the function called $\|\vartheta\|^{2}$ in [19]. 


$$
S^{\prime \prime}(n)=n\left(\tau_{2}+\tau_{1} \tau_{2}^{-1} \tau_{1}\right) n+2 i n\left(x-\tau_{1} \tau_{2}^{-1} y\right)
$$

Then the Poisson formula applied to $S^{\prime}$ gives

$$
\begin{gathered}
\sum_{\ell} \mathrm{e}^{-S^{\prime}(m=\ell+\mu, n)}=\sum_{j} \mathrm{e}^{2 \pi i j \mu} \int \mathrm{d} t \mathrm{e}^{-Q(t)} ; \\
Q(t) \equiv 2 \pi t \cdot \tau_{2}^{-1} \cdot t+2 \pi\left(i j-2 n \tau_{1} \tau_{2}^{-1}+2 i y \tau_{2}^{-1}\right) \cdot t .
\end{gathered}
$$

Performing the gaussian integral we get

$$
\begin{aligned}
Z_{\nu, \mu}= & \sum_{k, \ell} \mathrm{e}^{-S(n=k+\nu, m=\ell+\mu)} \\
= & 2^{g} E \sum_{k, j \in Z^{g}} \exp \left[i \pi\left(\left(k+\nu-\frac{1}{2} j\right) \tau\left(k+\nu-\frac{1}{2} j\right)-\left(k+\nu+\frac{1}{2} j\right) \bar{\tau}\left(k+\nu+\frac{1}{2} j\right)\right)\right. \\
& \left.\quad+4 \pi i\left(\frac{1}{2} j \cdot \mu+\frac{1}{2} i j \cdot y-(k+\nu) \cdot x\right)\right] \quad ; \\
E \equiv & 2^{-3 g / 2}\left(\operatorname{det} \tau_{2}\right)^{\frac{1}{2}} \mathrm{e}^{-2 \pi y \cdot \tau_{2}^{-1} \cdot y} .
\end{aligned}
$$

The "rotation" of the lattice mentioned above is accomplished as follows. We replace the sum over $k \in Z^{g}, \frac{1}{2} j \in \frac{1}{2} Z^{g}$ by a sum over all $\epsilon \in\left(Z_{2}\right)^{g}$ and $a, b \in Z^{g}$ such that $a \pm b$ has only even entries. The two lattices so defined are in 1-1 correspondence via

$$
k=\frac{1}{2}(a+b), \quad \frac{1}{2} j=\frac{1}{2}(b-a)+\epsilon .
$$

Also we can enforce the condition that $a \pm b$ be even by performing an unrestricted sum but including the $Z_{2}$ delta function:

$$
\delta(a-b \quad(\bmod 2))=2^{-g} \sum_{\zeta \in\left(\mathrm{Z}_{2}\right)^{g}} \mathrm{e}^{2 \pi i(a-b) \cdot \zeta} .
$$

Thus we have

$$
\begin{aligned}
Z_{\nu, \mu}=E \cdot \sum_{\substack{a, b \in Z^{a} \\
\epsilon, \zeta \epsilon\left(\mathbf{Z}_{2}\right)^{\theta}}} \exp [i \pi((a+\nu-\epsilon) r(a+\nu-\epsilon)-(b+\nu+\epsilon) \bar{\tau}(b+\nu+\epsilon)) \\
+2 \pi i((b-a+2 \epsilon)(\mu+i y)-(a+b+2 \nu) x+(a-b) \zeta)] \\
=E \cdot \sum \exp [i \pi(a+\nu-\epsilon) \tau(a+\nu-\epsilon)+2 \pi i((a+\nu-\epsilon)(-z-\mu+\zeta) \\
-i \pi(b+\nu+\epsilon) \bar{\tau}(b+\nu+\epsilon)-2 \pi i(b+\nu+\epsilon)(\bar{z}-\lambda+\varsigma)+4 \pi i \epsilon \cdot \zeta]
\end{aligned}
$$

Using the definition (2.42) we obtain (4.12). Note that we are permitted to change $\epsilon$ to $-\epsilon$ since $2 \epsilon$ is an integer vector. 


\section{Bosonic Correlations}

With (4.12) in hand we can now turn to the fluctuation part $\tilde{\varphi}$ of $\varphi$, and finally compare the bosonic correlation functions to (4.8).

$\widetilde{\varphi}$ has no jumps across the cuts of $\Sigma$, and so its action is given by (3.2), (3.8), and (3.13). Hence the right side of (4.4) is given by $Z_{\text {inst }}$ times

$$
\begin{aligned}
& Z_{\text {fluct }}=\int[\mathrm{d} \tilde{\varphi}] \exp \left[-\int_{\Sigma}\left(4 \pi i \partial \tilde{\varphi} \wedge \bar{\partial} \tilde{\varphi}+(1-2 \lambda) R_{K} \tilde{\varphi}\right)\right.
\end{aligned}
$$

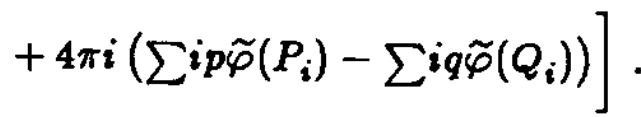

To do the gaussian integral change variables from $\tilde{\varphi}$ to

$$
\hat{\varphi}=\tilde{\varphi}-(2 \pi i)^{-1}\left[\sum \log G\left(P, P_{i}\right)-\sum \log G\left(P, Q_{i}\right)\right]
$$

and remove the zero mode from the integral over $\varphi$. Using (2.44)-(2.46) and integrating by parts several times we obtain

$$
Z_{\text {fluct }}=\left(\frac{\operatorname{det}^{\prime} \bar{\partial}^{\dagger} \bar{\partial}}{A_{\Sigma}}\right)^{-\frac{1}{2}} \cdot \frac{\prod_{i, j=1}^{p} G\left(P_{i}, P_{j}\right) \prod_{i, j=1}^{q} G\left(Q_{i}, Q_{j}\right)}{\prod G\left(P_{i}, Q_{j}\right)^{2}},
$$

where $A_{\Sigma}$ is the norm of the removed zero mode of $\bar{\partial}^{\dagger} \bar{\partial}$, i.e. the area of $\Sigma$ in the given metric. The simple form of (4.14) comes from the special normalization (2.46) chosen for the Green function. We have not used the Arakelov condition, however.

Of course as it stands (4.14) equals zero due to the coincident Green functions. We will define the path integral using zeta-function regulation on the determinant and the regulated coincident Green function (4.1). The freedom to make such a choice is the path-integral version of the freedom to choose a normal-ordering convention.

At last we can write out (4.4) in full detail, using (4.8), (4.13), and (4.14). Dropping an overall constant, the statement (4.4) of bosonization says that for spins $\lambda>1$,

$$
\begin{aligned}
& \frac{\operatorname{det}^{\prime} \bar{\partial}_{\mathcal{L}_{b}}^{\dagger} \bar{\partial}_{\mathcal{L}_{b}}}{\operatorname{det}\left(u_{i}, u_{j}\right)} \cdot\left\|\operatorname{det}\left(\begin{array}{cccccc}
u_{1}\left(P_{1}\right) & \cdots & u_{p-q}\left(P_{1}\right) & \mathcal{G}\left(P_{1}, Q_{1}\right) & \cdots & \mathcal{G}\left(P_{1}, Q_{q}\right) \\
\vdots & & \vdots & \vdots & & \vdots \\
u_{1}\left(P_{p}\right) & \cdots & u_{p-q}\left(P_{p}\right) & \mathcal{G}\left(P_{p}, Q_{1}\right) & \cdots & \mathcal{G}\left(P_{p}, Q_{q}\right)
\end{array}\right)\right\|^{2} \\
& =\left(\frac{\operatorname{det}^{\prime} \bar{\partial}^{\dagger} \bar{\partial}}{\operatorname{det}(i Y)^{-1} \cdot A_{\Sigma}}\right)^{-\frac{1}{2}} \cdot \mathcal{N}(z) \cdot \frac{\prod_{i, j=1}^{p} G\left(P_{i}, P_{j}\right) \prod_{i, j=1}^{q} G\left(Q_{i}, Q_{j}\right)}{\prod G\left(P_{i}, Q_{j}\right)^{2}}
\end{aligned}
$$


$\mathcal{N}$ is defined in (2.43), and $z$ is defined in (4.9).

For $\mathcal{L}_{b}=K$ we will give only the formula with no extra insertions, corresponding to (4.6). This case is interesting in that the same determinantal factor appears on both sides of (4.15), so that instead of relating two determinants we get a formula expressing one in terms of special functions on the Riemann surface:

$$
\left(\frac{\operatorname{det}^{\prime} \bar{\partial}^{\dagger} \bar{\partial}}{\operatorname{det}(i Y)^{-1} \cdot A_{\Sigma}}\right)^{3 / 2}=\left\|\operatorname{det} \omega^{i}\left(P_{j}\right)\right\|^{-2} \cdot \mathcal{N}(z) \cdot \frac{\prod_{i, j=1}^{g} G\left(P_{i}, P_{j}\right) \cdot G(Q, Q)}{\prod G\left(P_{i}, Q\right)^{2}}
$$

This formula can in turn be substituted in (4.15) to get formulæ for all the determinants.

Finally, for spin $\lambda=\frac{1}{2}$ generically there are no zero modes at all and we can take $p=q=0$. This gives

$$
\operatorname{det}^{\prime} \bar{\partial}_{\mathcal{L}_{b}}^{\dagger} \bar{\partial}_{\mathcal{L}_{b}}=\left(\frac{\operatorname{det}^{\prime} \bar{\partial}^{\dagger} \bar{\partial}}{\operatorname{det}(i Y)^{-1} \cdot A_{\Sigma}}\right)^{-\frac{1}{2}} \cdot \mathcal{N}\left(\mathcal{L}_{b} \otimes L_{A}^{-1}\right)
$$

the "spin- $\frac{1}{2}$ bosonization formula." This has already been derived (cf [6], [7]) and it forms the basis for our proof in the next section of (4.15) and (4.16). At one loop this formula is essentially the Jacobi triple product formula [25].

\section{Mathematical proof of bosonization}

We now present a mathematical proof of the bosonization formulæ obtained in the previous sections. We will prove (4.4) by proving its explicit restatements (4.15) and (4.16). Actually, for technical reasons we prove these identities only up to an overall constant depending on the genus, the spin, and the number of field insertions. This is adequate for proving the equivalence of two given field theories. For string applications, where one wants to relate different genera, factorization of amplitudes as a surface degenerates will fix the relative normalizations. Presumably the constants obtained in this way agree with the predictions of naive bosonization, but we will not enter into the analysis here.

\section{A. Weyl invariance}

As a first consistency check on (4.15) we now show that once it holds for any metric, it then holds for any conformally-related metric. This is a simple application of the conformal 
anomaly formula [50] [51] [52], which says that if $\widetilde{g}^{z \bar{z}}=\mathrm{e}^{2 \sigma} g^{z \bar{z}}$ then the zeta-regulated determinant behaves as

$$
\left[\frac{\operatorname{det}^{\prime} \bar{\partial}_{\mathcal{L}}^{\dagger} \bar{\partial}_{\mathcal{L}}}{\operatorname{det}\left(u_{i}, u_{j}\right) \cdot \operatorname{det}\left(v_{i}, v_{j}\right)}\right]^{\sim}=\exp \left[\frac{6 \lambda(\lambda-1)+1}{6 \pi i} S_{L}\right] \cdot \frac{\operatorname{det}^{\prime} \bar{\partial}_{\mathcal{L}}^{\dagger} \bar{\partial}_{\mathcal{L}}}{\operatorname{det}\left(u_{i}, u_{j}\right) \cdot \operatorname{det}\left(v_{i}, v_{j}\right)}
$$

Here $\left\{u_{i}\right\},\left\{v_{i}\right\}$ are zero modes of $\bar{\partial}_{\mathcal{L}}$ and $\bar{\partial}_{\mathcal{L}}^{\dagger}, \lambda$ is the spin of $\mathcal{L}$, and $S_{L}$ is defined in (2.49). We also have the result (2.48) on the rescaling of the Green function. (2.48) must however be modified for coincident points because of the regulator (4.1), which is not Weyl-invariant:

$$
\tilde{G}_{r}(P, P)=\exp \left[\frac{1}{4 \pi i(g-1)^{2}} S_{L}[\sigma]+\left(\frac{1}{g-1}+1\right) \sigma(P)\right] \cdot G_{r}(P, P)
$$

The net number of Green functions in (4.14) is $p^{2}+q^{2}-2 p q=(2 \lambda-1)^{2}(g-1)^{2}$ by (3.14). Collecting factors of $S_{L}$ and $\sigma\left(P_{i}\right)$ we therefore see that

$$
\begin{aligned}
& \tilde{Z}_{\text {fluct }}= \exp \left[-\frac{1}{12 \pi i} S_{L}+(2 \lambda-1)^{2}(g-1)^{2} \frac{1}{4 \pi i(g-1)^{2}} S_{L}\right] \\
& \cdot \exp \left[\frac{1}{2(g-1)}\left(2 p \sum \sigma\left(P_{i}\right)+2 q \sum \sigma\left(Q_{i}\right)-2 q \sum \sigma\left(P_{i}\right)-2 p \sum \sigma\left(Q_{i}\right)\right)\right. \\
&\left.+\sum \sigma\left(P_{i}\right)+\sum \sigma\left(Q_{i}\right)\right] \cdot Z_{\text {fluct }} \\
&= \exp \left[\frac{1}{6 \pi i}\left(6 \lambda^{2}-6 \lambda+1\right) S_{L}\right] \cdot \exp \left[2 \lambda \sum \sigma\left(P_{i}\right)+(2-2 \lambda) \sum \sigma\left(Q_{i}\right)\right] \cdot Z_{\text {fluct }}
\end{aligned}
$$

The Liouville part matches (5.1), while the remaining factor gives the correct rescaling properties of (4.3).

Thus we can require our metric to be in any convenient conformal slice. Only now will we use this freedom to choose the Arakelov metric, so that coincident Green functions equal one (eqn. (4.2)).

Had we used an alternate normal-ordering prescription to (4.1), we would have gotten coordinate-dependent factors and a different metric dependence at the $P_{i}, Q_{i}$. For example, in [5] we used a modification of (4.1) to get expressions for $b(P) \bar{b}(P)$, not (4.3). The two prescriptions are completely equivalent.

\section{B. Outline of proof}

The main ingredient in the proof is Quillen's treatment of a holomorphic family of Cauchy-Riemann operators on compact Riemann surfaces: the zeta-function regulated 
determinant $\operatorname{det}^{\prime} \bar{\partial}_{\xi}^{\dagger} \bar{\partial}_{\xi}$ is used to define a metric, the "Quillen metric" on the determinant line bundle of $\xi$. The bosonization formulæ can be seen as asserting that some natural isomorphisms of determinant line bundles are isometries when one uses the Quillen metrics. We will establish these isometries from two basic results:

1. The spin-1/2 bosonization formula (4.17). Recall that in this formula $\mathcal{L}_{b}$ is any twisted spin bundle, i.e. any line bundle on $\Sigma$ of degree $g-1 . \quad \Sigma$ is equipped with an arbitrary metric. This metric in turn defines a metric on the preferred spin bundle $L_{A}$ (the one which makes the isomorphism $L_{A}^{2} \cong K$ an isometry), and hence a metric on $\mathcal{L}_{b}$. The LHS of (4.17) is computed using this metric. On the RHS $(i Y)^{-1}$ is the period matrix of $\Sigma, A_{\Sigma}$ is the metric area of $\Sigma$, and $\mathcal{N}$ is defined in (2.43).

We note that all of the bosonization formulæ involve the same function $(\cdots)^{-\frac{1}{2}} \mathcal{N}$, which is essentially the spin-1/2 determinant [6][7]. Thus it seems that one could prove all the results we need by referring each spin to the known case of spin $1 / 2$. This is accomplished by

2. The insertion theorem, given below in subsection $D$, which relates $\operatorname{det}^{\prime} \bar{\partial}_{\xi}^{\dagger} \bar{\partial}_{\xi}$ and $\operatorname{det}^{\prime} \bar{\partial}_{\xi \otimes O(P)}^{\dagger} \bar{\partial}_{\xi \otimes O(P)}$ for any line bundle $\xi$ and any point $P$ on $\Sigma$. It is in this theorem that the Arakelov metric plays a simplifying role.

The insertion theorem is the mathematical counterpart of the insertions of $b(P)$ or $c(Q)$ in the functional determinants of section four. It is also closely related to the third axiom defining metrics on direct image bundles in Faltings' work on arithmetic geometry $[19]$. In fact, this theorem allows one to prove that the norm used by Faltings differs from the Quillen norm only by a multiplicative factor depending only on the surface $\Sigma[52]$.

We will prove formulæ slightly more general than the bosonization identities of section four. Indeed, we consider not only the line bundles $\mathcal{L}_{b}$ with degree a multiple of $g-1$, but arbitrary line bundles $\xi$ on $\Sigma$ of degree $d \geq g-1$. In general $\xi$ does not have an Arakelov metric. Instead we will demand that the metric on $\xi$ be "admissible," which means that its curvature is proportional to the Arakelov curvature form $\mu_{\mathrm{Arak}}$ in (2.53). Thus the Arakelov metric itself is admissible. Admissible metrics always exist; they are unique up to a constant since $\Sigma$ is compact. 
To state our more general formula let us begin by supposing that

$$
H^{1}(\Sigma ; \xi)=0
$$

Recall from (2.16) that this condition means that the adjoint $\bar{\partial}_{\xi}^{\dagger}$ has no zero mode. This statement is independent of the metrics chosen on $\Sigma$ and $\xi$. It is satisfied if $d>2 g-2$ or if $d=2 g-2$ but $\xi \not K$, or if $d=g-1$ and $\xi$ does not belong to the theta divisor. We will sketch later the necessary modification to the proof when (5.2) is not satisfied for example in the proof of (4.16).

We also have from (2.10) that $\operatorname{dim} H^{\circ}(\sigma ; \xi)=\operatorname{dim} \operatorname{ker} \bar{\partial}_{\xi} \equiv k$. Using the simplifying condition (5.2) and the Riemann-Roch theorem (2.18) we then get that $k=d+1-g$. Let $u_{1}, \ldots, u_{k}$ be a basis of $H^{0}(\Sigma ; \xi)$.

For any integer $q \geq 0$, let $p=q+k$. Suppose we are given $p+q$ pairwise distinct points on $\Sigma, P_{1}, \ldots, P_{p}, Q_{1}, \ldots, Q_{q}$. From these points we can build a determinant generalizing the one in (4.8):

$$
\operatorname{Det}\left(u_{i}, P_{j}, Q_{\ell}\right) \equiv \operatorname{det}\left(\begin{array}{cccccc}
u_{1}\left(P_{1}\right) & \cdots & u_{k}\left(P_{1}\right) & \mathcal{G}\left(P_{1}, Q_{1}\right) & \cdots & \mathcal{G}\left(P_{1}, Q_{q}\right) \\
\vdots & & \vdots & \vdots & & \vdots \\
u_{1}\left(P_{p}\right) & \cdots & u_{k}\left(P_{p}\right) & \mathcal{G}\left(P_{p}, Q_{1}\right) & \cdots & \mathcal{G}\left(P_{p}, Q_{q}\right)
\end{array}\right)
$$

The parametrix $\mathcal{G}$ is again defined by (4.7), where now $\mathcal{L}_{b}$ is replaced by the arbitrary line bundle $\xi .{ }^{12}$ Again the residue of $\mathcal{G}$ as $P \rightarrow Q$ is $1 / 2 \pi i$. $\operatorname{Det}\left(u_{i}, P_{j}, Q_{\ell}\right)$ is an element of $\left[\left.\otimes_{i=1}^{p} \xi\right|_{P_{i}}\right] \otimes\left[\left.\otimes_{j=1}^{q}\left(\xi^{-1} \otimes K\right)\right|_{Q_{j}}\right]$.

We also suppose that $\Sigma$ is equipped with the Arakelov metric, and that $\xi$ is equipped with an admissible metric.

The generalization of (4.15) which we will prove then says that with the above choices, when (5.2) is satisfied we have

$$
\begin{aligned}
\left\|\operatorname{Det}\left(u_{i}, P_{j}, Q_{\ell}\right)\right\|^{2} \cdot \frac{\operatorname{det}^{\prime} \bar{\partial}_{\xi}^{\dagger} \bar{\partial}_{\xi}}{\operatorname{det}\left(u_{i}, u_{j}\right)} \\
=A(g, d, q)\left(\frac{\operatorname{det}^{\prime} \bar{\partial}^{\dagger} \bar{\partial}}{\operatorname{det}(i Y)^{-1} \cdot A_{\Sigma}}\right)^{-\frac{1}{2}} \cdot \mathcal{N}\left(\xi \otimes O\left(-D_{i n s}\right) \otimes L_{A}^{-1}\right) \\
\times \frac{\prod_{i<j} G\left(P_{i}, P_{j}\right)^{2} \prod_{i<j} G\left(Q_{i}, Q_{j}\right)^{2}}{\prod G\left(P_{i}, Q_{j}\right)^{2}}
\end{aligned}
$$

12 Again when (5.2)is not satisfied we replace the unit operator in (4.7) by the projector off the zero modes of $\bar{\partial}_{\xi}^{\dagger}$. 
where $A(g, d, q)$ is a constant which depends only on $g, d$, and $q$, and

$$
D_{i n s}=\sum_{i=1}^{p} P_{i}-\sum_{i=1}^{q} Q_{i} \text {. }
$$

Taking $\xi=\mathcal{L}_{b}$ gives (4.15). This follows because with the Arakelov norm coincident Green functions vanish, while each noncoincident function in the numerator of (4.15), (4.16) appears twice.

The proof of (5.4) will go roughly as follows. Thanks to the insertion theorem applied $p+q$ times, we can relate $\operatorname{det}^{\prime} \bar{\partial}_{\xi}^{\dagger} \bar{\partial}_{\xi}$ and $\operatorname{det}^{\prime} \bar{\partial}_{\xi^{\prime}}^{\dagger} \bar{\partial}_{\xi^{\prime}}$ where $\xi^{\prime}=\xi \otimes O\left(-D_{i n s}\right)$. Next, as the degree of $\xi^{\prime}$ is $d+q-p=d-k=g-1$, we can relate $\operatorname{det}^{\prime} \bar{\partial}_{\xi^{\prime}}^{\dagger} \bar{\partial}_{\xi^{\prime}}$ to $\operatorname{det}^{\prime} \bar{\partial}^{\dagger} \bar{\partial}$ by the spin-1/2 bosonization formula. The finite dimensional determinants occur in (5.4) because the precise definitions of the determinant line bundle and of the Quillen metric on it involve the finite dimensional spaces of zero modes of $\bar{\partial}$ operators and their adjoints, so that we have to take care of them when we apply the insertion theorem. The bosonic Green functions occur because this theorem makes essential use of the admissible metrics on the $O(P)$ 's defined by (2.50).

We can also derive formulæ analogous to (5.4) when the condition (5.2) is not satisfied. We will only consider the case $\xi=K$, equipped with the Arakelov metric. Again let $q>0$ be an integer, let $p=g-1+q$, and let $P_{1}, \ldots, P_{p}, Q_{1}, \ldots, Q_{q}$ be pairwise distinct points of $\Sigma$. We can then build the determinant

$$
\operatorname{Det}\left(\omega_{i}, P_{j}, Q_{\ell}\right) \equiv \operatorname{det}\left(\begin{array}{cccccc}
\omega_{1}\left(P_{1}\right) & \cdots & \omega_{2 g-2}\left(P_{1}\right) & \mathcal{G}\left(P_{1}, Q_{1}\right) & \cdots & \mathcal{G}\left(P_{1}, Q_{q}\right) \\
\vdots & & \vdots & \vdots & & \vdots \\
\omega_{1}\left(P_{p}\right) & \cdots & \omega_{2 g-2}\left(P_{p}\right) & \mathcal{G}\left(P_{p}, Q_{1}\right) & \cdots & \mathcal{G}\left(P_{p}, Q_{q}\right) \\
0 & \cdots & 0 & 1 & \cdots & 1
\end{array}\right) .
$$

It belongs to $\left.\bigotimes_{i=1}^{g-1+q} K\right|_{P_{i}}$.

Then we have the equality

$$
\begin{gathered}
\left\|\operatorname{Det}\left(\omega_{i}, P_{j}, Q_{\ell}\right)\right\|^{2} \cdot \frac{\operatorname{det}^{\prime} \bar{\partial}_{K}^{\dagger} \bar{\partial}_{K}}{(1,1) \cdot \operatorname{det}\left(\omega_{i}, \omega_{j}\right)} \\
=B(g, q)\left(\frac{\operatorname{det}^{\prime} \bar{\partial}^{\dagger} \bar{\partial}}{\operatorname{det}(i Y)^{-1} \cdot A_{\Sigma}}\right)^{-\frac{1}{2}} \cdot \mathcal{N}\left(O\left(-D_{i n s}\right) \otimes L_{\mathfrak{A}}\right) \\
\times \frac{\prod_{i<j} G\left(P_{i}, P_{j}\right)^{2} \prod_{i<j} G\left(Q_{i}, Q_{j}\right)^{2}}{\prod G\left(P_{i}, Q_{j}\right)^{2}}
\end{gathered}
$$


where $B(g, q)$ is a constant depending only on $g, q$. When $q=1$ we then recover (4.16). (See the comments surrounding (4.6).)

Strictly speaking in this paper we will prove (5.4) and (5.6) only for $g>2 .{ }^{13}$ To get complete proofs when $g \leq 2$, one needs estimates on the growth of regularized determinants and Green functions when $\Sigma$ degenerates into a Riemann surface with one node, which we will not discuss here.

Note that (5.4) and (5.6) are closely related to some classical identities in the theory of abelian functions on Riemann surface [53], in particular the trisecant identity [23][22].

\section{The local Riemann-Roch theorem}

In this subsection we review some basic facts about determinant line bundles, Quillen metrics, holomorphic families of $\bar{\partial}$ operators on compact Riemann surface, and the Riemann-Roch theorem for families (cf [13], [54], [17], [18]).

If $D$ is an elliptic differential operator on a compact manifold, one defines the one dimensional vector space

$$
\operatorname{DET} D=\left(\wedge^{\max } \operatorname{ker} D\right)^{-1} \otimes\left(\wedge^{\max } \operatorname{coker} D\right)
$$

Formally DET $D$ is the dual of the "top exterior power" of the family index of $D$.

It is important for our purposes to consider not only one particular $D$ but a parametrized family of operators. We therefore need a notion of a family $\left\{\Sigma_{s}\right\}$ of Riemann surfaces, with a family of line bundles $\left\{\xi_{s}\right\}$ on them. We can glue together all the Riemann surfaces into a large space $X$, and glue the $\xi_{a}$ into a single bundle $E$ over the total space $X$. In this paper we will actually consider holomorphic families of Riemann surface and bundles. Thus we let $\pi: X \rightarrow S$ be a proper holomorphic submersion, the fibers of which are compact and of complex dimension one. We also take $E$ to be a holomorphic vector bundle on $X$ and $F=E \otimes \bar{K}_{X \mid S}$, where $K_{X \mid S}$ is the line bundle of vertical $(1,0)$-forms on $X$. For each $s \in S$ we then get an elliptic operator $\bar{\partial}_{s}: C^{\infty}\left(\Sigma_{s} ; \xi_{s}\right) \rightarrow C^{\infty}\left(\Sigma_{s} ; \xi_{\theta} \otimes \bar{K}\right)$ on the Riemann surface $\Sigma_{s}=\pi^{-1}(s) . \bar{\partial}_{s}$ is called the Cauchy-Riemann operator "coupled to $\xi_{s}$ " and the family so defined is denoted by $\bar{\partial}_{E}$. The determinant line bundle DET $\bar{\partial}_{E}$ has a canonical holomorphic structure [55][17].

\footnotetext{
${ }^{13}$ See lemma 2 below.
} 
This construction was introduced first within the framework of algebraic geometry [56], [55]; the construction for smooth families appears e.g. in [57], [54].

One can define a norm on the determinant line bundle as follows. Suppose we are given a smooth family of riemannian metrics on the fibers $\Sigma_{s}$ (i.e. a smooth metric on the vertical tangent bundle $K_{X \mid S}^{-1}$ ), and a smooth hermitian metric on $E$. Then for any $s \in S, \bar{\partial}_{s}^{\dagger}$ is defined, and ker $\bar{\partial}_{s}$ and coker $\bar{\partial}_{s}$ have natural $L^{2}$ metrics, which define a metric $\|\cdot\|_{L^{2}}$ on DET $\bar{\partial}_{s}$. Generally, because of the jumps of the dimension of ker $\bar{\partial}_{\theta}$, this norm is not smooth on all of $S$. However, the "Quillen norm"

$$
\|\cdot\|_{Q}^{2} \equiv \operatorname{det}^{\prime} \bar{\partial}_{s}^{\dagger} \bar{\partial}_{s} \cdot\|\cdot\|_{L^{2}}^{2}
$$

is always a smooth metric on $\operatorname{DET} \bar{\partial}_{E}[13][54]$.

We can now state a local Riemann-Roch theorem for families of curves. Recall that for any hermitian metric on a holomorphic vector bundle like $E$ there is a unique unitary connectiois on $E$ compatible with its holomorphic structure. Using this connection and the Che $\mathrm{n}$-Weil formulæ for characteristic classes, we can associate to a line bundle $E$ and its metric the Chern forms $c_{1}\left(E,\|\cdot\|_{E}\right)$, the Chern character form $\mathrm{Ch}\left(E,\|\cdot\|_{E}\right)$, and the Todd form $\operatorname{Td}\left(E,\|\cdot\|_{E}\right)$ using the polynomials $\operatorname{Ch}(x)=\mathrm{e}^{x}, \operatorname{Td}(x)=1+\frac{1}{2} x+\frac{1}{12} x^{2}+\cdots$. It is important to note that in the family setting these forms are constructed from the full curvature of the bundle $E$ over $X$, not just its vertical parts. If $\sigma$ is any differential form, we denote by $\sigma^{(k)}$ its component of degree $k$.

Theorem. Let $\pi: X \rightarrow S$ be a holomorphic family of Riemann surfaces and $E$ a holomorphic vector bundle over $X$. Let $\|\cdot\|$ be any smooth hermitian metric on the tangent bundle $K_{X \mid S}^{-1}$ and $\|\cdot\|_{E}$ a smooth hermitian metric on $E$. Let $\|\cdot\|_{Q}$ be the Quillen metric they define on DET $\bar{\partial}_{E}$. Then one has the formula

$$
\mathrm{c}_{1}\left(\operatorname{DET} \bar{\partial}_{E},\|\cdot\|_{Q}\right)=-\int_{X \mid S}\left\{\operatorname{Ch}\left(E,\|\cdot\|_{E}\right) \wedge \operatorname{Td}\left(K_{X \mid S}^{-1},\|\cdot\|\right)\right\}^{(4)} \text {. }
$$

Here $\int_{X \mid S}$ denotes integration of a form along the fibers $\Sigma$ of $\pi$. Note that (5.9) makes no use of the admissibility condition; it works for any metrics.

The cohomological form of (5.9) is a direct consequence of the Atiyah-Singer index theorem for families, or of the Riemann-Roch-Grothendieck theorem (which gives a more 
precise formula, true in the rational Chow group of $S$ ). The formula (5.9) was proved by Quillen [13] when $X=X_{0} \times S$ and the metrics are fixed, and by Belavin and Knizhnik [14] when $E=K^{n}$. Bismut and Freed have proven an analogous statement for families of Dirac operators [54], from which one can deduce the general formula [15][18].

\section{The insertion theorem}

Let $\Sigma$ be a compact connected Riemann surface of genus $g>0$, equipped with its Arakelov metric, and let $\xi$ be an arbitrary line bundle on $\Sigma$, equipped with an admissible metric. For any point $P$ of $\Sigma$, we get an admissible metric on $\xi \otimes O(-P)$ by multiplying the given metric on $\xi$ by the canonical metric on $O(-P)$, i.e. the metric dual to the metric on $O(P)$ given by (2.50). From these data, we obtain Quillen metrics on the one-dimensional spaces DET $\bar{\partial}_{\xi}$ and DET $\bar{\partial}_{\xi \otimes O(-P)}$.

On the other hand, the long exact sequence

$$
\left.0 \rightarrow H^{0}(\Sigma ; \xi \otimes O(-P)) \rightarrow H^{0}(\Sigma ; \xi) \rightarrow \xi\right|_{P} \rightarrow H^{1}(\Sigma ; \xi \otimes O(-P)) \rightarrow H^{1}(\Sigma ; \dot{\xi}) \rightarrow 0
$$

associated to (2.8) gives rise to a canonical isomorphism of one dimensional vector spaces, by taking the top exterior power:

$$
I:\left.\operatorname{DET} \bar{\partial}_{\xi \otimes O(-P)} \cong\left(\mathrm{DET} \bar{\partial}_{\xi}\right) \otimes \xi\right|_{P}
$$

We have used (2.16) to replace the cokernel in (5.7) by $H^{1}$.

Insertion theorem. The isomorphism (5.10) is an isometry when $\xi_{P}$ has the given metric and the determinant spaces are given the Quillen metrics (up to a multiplicative constant depending only on the genus of $\Sigma$ and on the degree of $\xi$ ).

For $g>2$, the theorem is a direct consequence of the following lemmas:

Lemma 1. Let $\pi: X \rightarrow S$ be a holomorphic family of compact connected Riemann surfaces of genus $g>0, E$ a holomorphic line bundle on $X$, and $\sigma: S \rightarrow X$ a holomorphic section of $\pi$. Let $\Sigma_{s}=\pi^{-1}(s)$ and $\xi_{s}=\left.E\right|_{\Sigma_{s}}$.

i) The family consisting of the Arakelov metrics on the Riemann surfaces $\Sigma_{s}$ defines a smooth metric on the vertical tangent bundle $K_{X \mid S}^{-1}$. 
ii) The family of canonical metrics (2.50) on the line bundles $O(-\sigma(s))$ over $\Sigma_{s}$, $s \in S$, defines a smooth metric on $O\left(-\sigma(S)\right.$ ) (note that $\left.O(-\sigma(S))\right|_{\Sigma} \cong$ $O(-\sigma(s)))$.

iii) The family of isomorphisms

$$
I_{\theta}:\left.\operatorname{DET} \bar{\partial}_{\xi_{\theta} \otimes O(-\sigma(s))} \cong\left(\operatorname{DET} \bar{\partial}_{\xi_{0}}\right) \otimes \xi_{s}\right|_{\sigma(s)}
$$

(cf (5.10)) defines an isomorphism of holomorphic line bundles on $S$

$$
I: \operatorname{DET} \bar{\partial}_{E^{\prime}} \cong \operatorname{DET} \bar{\partial}_{E} \otimes \sigma^{*}(E)
$$

where $E^{\prime}=E \otimes O(-\sigma(S))$.

iv) Suppose that $E$ is equipped with a smooth metric $\|\cdot\|_{E}$ whose restriction to any $\Sigma_{s}$ is an admissible metric on $\xi_{s}$, and that $K_{X \mid S}$ and $O(-\sigma(S))$ are equipped with the Arakelov metric and the canonical metric defined in (i) and (ii). Using these metrics, we obtain Quillen metrics $\|\cdot\|_{Q}$ and $\|\cdot\|_{Q}^{\prime}$ on the line bundles DET $\bar{\partial}_{E}$ and DET $\bar{\partial}_{E^{\prime}}$ on $S$. Then we have the equality of differential forms

$$
\mathrm{c}_{1}\left(\mathrm{DET} \bar{\partial}_{E^{\prime}},\|\cdot\|_{Q}^{\prime}\right)=\mathrm{c}_{1}\left(\mathrm{DET} \bar{\partial}_{E},\|\cdot\|_{Q}\right)+\sigma^{*} \mathrm{c}_{1}\left(E,\|\cdot\|_{E}\right)
$$

Hence when $S$ is compact and connected, the isomorphism $I$ in (5.11) is an isometry up to an overall constant. The next lemma says that for $g>2$ we can always take $S$ to be compact and connected.

Lemma 2. Let $\Sigma_{0}, \Sigma_{1}$ be two compact connected Riemann surfaces with the same genus $g>2$ and let $\xi_{0}, \xi_{1}$ be holomorphic line bundles on them with the same degree d. Suppose each $\xi_{i}$ is equipped with an admissible metric $\|\cdot\|_{i}$ and a point $P_{i}$. There exists a compact and connected complex manifold $S$, a holomorphic family $\pi: X \rightarrow S$ of compact connected Riemann surfaces, and a holomorphic line bundle $E$ on $X$ equipped with a smooth metric $\|\cdot\|_{E}$ which, restricted to any $\Sigma_{s}=\pi^{-1}(s)$, is admissible, and two points $s_{i} \in S, i=1,2$ and two isomorphisms $\varphi_{i}: \Sigma_{i} \rightarrow \Sigma_{s_{i}}$ such that

$$
\left(\xi_{i},\|\cdot\|_{i}\right) \cong \varphi_{i}^{*}\left(E,\|\cdot\|_{E}\right)
$$


Lemma 2 is an easy consequence of the hard fact that, for $g>2$, for any two points in the moduli space of smooth irreducible curves $\mathcal{M}_{g}$ there exists a complete curve which contains those two points [58].

The first two assertions of Lemma 1 are consequences of the definitions and of the theory of families of elliptic operators. The third assertion is a consequence of the definition of the holomorphic structure on the determinant line bundle. We will not enter here into the details of the proof of these assertions. The fourth assertion is a consequence of the local Riemann-Roch theorem of subsection $\mathrm{C}$ and the choice of Arakelov (resp. admissible) metrics on $K, \xi$. We now give the proof of the equality (5.12).

Let $D$ denote the hypersurface $\sigma(S)$, and let $[D]$ be the current, or form-valued distribution, associated to $D$. That is, for any form $\omega$ of degree the real dimension of $D$ one has $\int_{X}[D] \omega=\int_{D} \omega$.

Let $\widetilde{G}_{D}(x)=\left\|1_{O(D)}\right\|(x)$, where $O(D)$ is equipped with the canonical metric. The function $\tilde{G}_{D}$ is smooth on $X-D$, and it vanishes on $D$. If $G$ is the Arakelov Green function on $\Sigma_{s}$, we have

$$
\widetilde{G}_{D}(x)=G(x, \sigma \circ \pi(x))
$$

Furthermore, the following statements are easily proved:

a) The first Chern form of the line bundle $O(-D)$ equipped with the canonical metric satisfies the following equality:

$$
c_{1}(O(-D),\|\cdot\|)=-\frac{1}{2 \pi i} \partial \bar{\partial} \log \widetilde{G}_{D}-[D]
$$

(See the remark after (2.27).) The RHS is the sum of two non-smooth currents.

b) For any smooth differential form $\omega$ on $X$

$$
\int_{X \mid S}[D] \omega=\sigma^{*} \omega
$$

c) For any closed differential form $\omega$ of type $(1,1)$ on $X$, and for any distribution $\phi$ on $X$,

$$
\int_{X \mid S}(\partial \bar{\partial} \phi) \omega=\partial \bar{\partial} \int_{X \mid S} \phi \omega
$$

The equality (5.12) now follows from the following computation of first Chern forms, where we do not write explicitly the metrics on the various line bundles (they are the 
metrics specified in the statement of Lemma 1):

$$
\begin{aligned}
\mathrm{c}_{1}\left(\operatorname{DET} \bar{\partial}_{E}\right)-\mathrm{c}_{1}\left(\operatorname{DET} \bar{\partial}_{E^{\prime}}\right) & =-\int_{X \mid S}\left\{\left[\operatorname{Ch} E-\operatorname{Ch} E^{\prime}\right] \operatorname{Td} K_{X \mid S}^{-1}\right\}^{(4)} \\
& =-\int_{X \mid S}\left\{[1-\operatorname{Ch}(O(-D))] \operatorname{Ch} E \operatorname{Td} K_{X \mid S}^{-1}\right\}^{(4)} \\
& =\int_{X \mid S} c_{1}(O(-D))\left[c_{1}(E)-\frac{1}{2} c_{1}\left(K_{X \mid S}\right)+\frac{1}{2} c_{1}(O(-D))\right]
\end{aligned}
$$

The first equality follows from the local Riemann-Roch theorem, the second from the multiplicativity of the Chern character, and the last from the expressions for $\mathrm{Ch}$ and $\mathrm{Td}$. Using (5.14), we can rewrite the integral (5.15) as the sum of

$$
\omega_{1}=-\int_{X \mid S} \frac{1}{2 \pi i} \partial \bar{\partial} \log \widetilde{G}_{D} \cdot\left[c_{1}(E)-\frac{1}{2} c_{1}\left(K_{X \mid S}\right)+\frac{1}{2} c_{1}(O(-D))\right]
$$

and

$$
\omega_{2}=-\int_{X \mid S}[D] \cdot\left[c_{1}(E)-\frac{1}{2} c_{1}\left(K_{X \mid S}\right)+\frac{1}{2} c_{1}(O(-D))\right] .
$$

The identity (5.16) shows that $\omega_{1}=\partial \bar{\partial} F$, where

$$
f=-\frac{1}{2 \pi i} \int_{X \mid S} \log \tilde{G}_{D} \cdot\left[\mathrm{c}_{1}(E)-\frac{1}{2} \mathrm{c}_{1}\left(K_{X \mid S}\right)+\frac{1}{2} \mathrm{c}_{1}(O(-D))\right] .
$$

The restriction of the quantity in brackets to any $\Sigma_{s}$ is a multiple of $\mu_{\text {Arak }}$, thanks to the admissibility hypothesis on the metrics on $E, K_{X \mid S}$, and $O(-D)$. Next the formula (5.13) and the normalization condition (2.46) show that $F \equiv 0$. Hence $\omega_{1}=0$. On the other hand, the identity (5.15) shows that

$$
\omega_{2}=-\sigma^{*} \mathrm{c}_{1}(E)+\frac{1}{2} \mathrm{c}_{1}\left(\sigma^{*}\left(K_{X \mid S} \otimes O(D)\right)\right) .
$$

Recall that the Arakelov metric and the canonical metric are such that the residue map (2.52) is an isometry. This implies that the line bundle with metric $\sigma^{*}\left(K_{X \mid S} \otimes O(D)\right)$ is canonically isomorphic to the trivial bundle on $S$, with the trivial metric. Hence its first Chern form is zero, and $\omega_{2}=-\sigma^{*} c_{1}(E)$.

Finally we get

$$
c_{1}\left(\operatorname{DET} \bar{\partial}_{E}\right)-c_{1}\left(\operatorname{DET} \bar{\partial}_{E^{\prime}}\right)=\omega_{1}+\omega_{2}=-\sigma^{*} c_{1}(E)
$$

as was to be proved.

Having established (5.12) we now invoke Lemma 2 to say that metrics with the same curvature on $S$ must be equal up to a constant. This establishes the insertion theorem. 


\section{E. Proof of (5.4)}

Using the spin-1/2 bosonization formula (4.17) we see that the desired formula (5.4) follows from the following equality:

$\left\|\operatorname{Det}\left(u_{i}, P_{j}, Q_{\ell}\right)\right\|^{2} \cdot \frac{\operatorname{det}^{\prime} \bar{\partial}_{\xi}^{\dagger} \bar{\partial}_{\xi}}{\operatorname{det}\left(u_{i}, u_{j}\right)}=C(g, d, q) \frac{\prod_{i<j} G\left(P_{i}, P_{j}\right)^{2} \prod_{i<j} G\left(Q_{i}, Q_{j}\right)^{2}}{\prod G\left(P_{i}, Q_{j}\right)^{2}} \cdot \operatorname{det}^{\prime} \bar{\partial}_{\xi^{\prime}}^{\dagger} \bar{\partial}_{\xi^{\prime}} \cdot$

when $\xi^{\prime} \equiv \xi \otimes \mathcal{O}\left(-D_{\text {ins }}\right)$ is equipped with the product of the given admissible metric on $\xi$ and the canonical metric on $O\left(-D_{i n \theta}\right)$. Again $D_{i n s}$ is the divisor of insertion points (3.23). To prove (5.17) we use the insertion theorem.

Consider the following short exact sequence of sheaves ( $c f(2.8))$ :

$$
\left.0 \rightarrow \zeta \otimes O\left(-\sum P_{i}\right) \rightarrow \varsigma \rightarrow \zeta\right|_{\sum P_{i}} \rightarrow 0
$$

Taking $\varsigma=\xi \otimes O\left(\sum Q_{i}\right)$ we get

$$
\left.0 \rightarrow \xi \otimes O\left(-D_{i n 8}\right) \rightarrow \xi \otimes O\left(\sum Q_{i}\right) \rightarrow\left[\xi \otimes O\left(\sum Q_{i}\right)\right]\right|_{\sum P_{i}} \rightarrow 0
$$

Setting all the $Q_{i}=P_{i}$ we get

$$
\left.0 \rightarrow \xi \rightarrow \xi \otimes O\left(\sum Q_{i}\right) \rightarrow\left[\xi \otimes O\left(\sum Q_{i}\right)\right]\right|_{\sum Q_{i}} \rightarrow 0
$$

From the cohomology long exact sequences associated to these short exact sequences we deduce canonical isomorphisms of the one-dimensional vector spaces ( $c f(5.10)$ ):

$$
\begin{aligned}
I_{1}: \operatorname{DET} \bar{\partial}_{\xi \otimes O\left(-D_{i n}\right)} & \stackrel{\sim}{\longrightarrow} \operatorname{DET} \bar{\partial}_{\xi \otimes O}\left(\sum Q_{j}\right) \\
& \cong \bigwedge^{p}\left(\left.\bigoplus_{j=1}^{p}\left(O\left(\sum_{i=1}^{q} Q_{i}\right)\right)\right|_{P_{j}}\right) \\
I_{2}: \operatorname{DET} \bar{\partial}_{\xi \otimes O} \bar{\partial}_{\xi} \stackrel{\sim}{\sim} \operatorname{DET} \bar{\partial}_{\xi \otimes O}\left(\sum Q_{j}\right) & \otimes\left[\left.\bigotimes_{i=1}^{p} \xi\right|_{P_{i}}\right] \\
& \cong \operatorname{DET} \bar{\partial}_{\xi \otimes O\left(\sum Q_{j}\right)} \otimes\left[\left.\bigoplus_{j=1}^{q}\left(O\left(\sum_{i=1}^{q} Q_{i}\right)\right)\right|_{Q_{j}}\right)
\end{aligned}
$$


In the second lines of $(5.21),(5.22)$ we have used the unit section to trivialize $O\left(\sum Q_{i}\right)$ away from the $Q_{i}$. In the second line of (5.22) we have used the canonical isomorphism (2.52).

From $I_{1}$ and $I_{2}$ one can build an isomorphism

$$
I: \operatorname{DET} \bar{\partial}_{\xi} \stackrel{\sim}{\longrightarrow} \operatorname{DET} \bar{\partial}_{\xi^{\prime}} \otimes\left[\left.\bigotimes_{i}^{p} \xi\right|_{P_{i}}\right] \otimes\left[\left.\bigotimes_{1}^{q}\left(\xi^{-1} \otimes K\right)\right|_{Q_{j}}\right] .
$$

Then we have the general insertion formula, when the determinant bundles are equipped with the Quillen metrics, $\xi$ with the given admissible metric, and $K$ with the Arakelov metric:

$$
\|I(v)\|=D(g, d, p, q) \cdot \frac{\prod_{i<j} G\left(P_{i}, P_{j}\right) \prod_{i<j} G\left(Q_{i}, Q_{j}\right)}{\prod G\left(P_{i}, Q_{j}\right)} \cdot\|v\| .
$$

This formula is true for any $\xi$ and any collection $\left\{P_{1}, \ldots, P_{p}, \ldots, Q_{q}\right\}$ of pairwise distinct points on $\Sigma$; the conditions (5.2) and $p=q+d+1-g$ do not matter here.

The general insertion formula (5.23) follows from the insertion theorem by induction on $p, q$. Hence it too relies on the Arakelov condition. We now give the details for the case $p=2, q=0$ to show how the bosonic Green function enters.

The isomorphism (5.21) in this case reads

$$
I_{1}:\left.\left.\operatorname{DET} \bar{\partial}_{\xi \otimes O\left(-P_{1}-P_{2}\right)} \stackrel{\sim}{\longrightarrow} \mathrm{DET} \bar{\partial}_{\xi} \otimes \xi\right|_{P_{1}} \otimes \xi\right|_{P_{2}}
$$

It is obtained by composition and tensor product from the "insertion isomorphisms"

$$
\begin{aligned}
\operatorname{DET} \bar{\partial}_{\xi \otimes O\left(-P_{1}\right)} & \left.\cong \operatorname{DET} \bar{\partial}_{\xi} \otimes \xi\right|_{P_{1}} \\
\operatorname{DET} \bar{\partial}_{\xi \otimes O\left(-P_{1}-P_{2}\right)} & \left.\cong \operatorname{DET} \bar{\partial}_{\xi \otimes O\left(-P_{1}\right)} \otimes\left(\xi \otimes O\left(-P_{1}\right)\right)\right|_{P_{2}}
\end{aligned}
$$

and from the canonical isomorphism

$$
\begin{aligned}
\left.\left.\left(\xi \otimes O\left(-P_{1}\right)\right)\right|_{P_{2}} \cong \xi\right|_{P_{2}} \\
v \otimes 1_{O\left(-P_{1}\right)}\left(P_{2}\right) \mapsto v
\end{aligned}
$$

By the insertion theorem, (5.24a) and (5.24b) are isometries up to constants. On the other hand, (5.24c) multiplies the norms by $\left\|1_{O\left(-P_{1}\right)}\left(P_{2}\right)\right\|^{-1}=G\left(P_{1}, P_{2}\right)$. So the isomorphism $I$ multiplies the norms by (a constant times) $G\left(P_{1}, P_{2}\right)$.

Proceeding in this way, and using (5.22) we obtain (5.23). 
Now we can complete the proof of (5.17). It is enough to prove this formula when

$$
H^{0}\left(\Sigma ; \xi \otimes O\left(-D_{\text {ins }}\right)\right)=0
$$

i.e. when $\xi^{\prime}=\xi \otimes O\left(-D_{i n s}\right)$ does not belong to the theta divisor. Indeed this condition is satisfied for a generic choice of insertion points since $H^{1}(\Sigma ; \xi)=0$. Moreover, one can see directly that when (5.25) is not satisfied, the two sides of (5.4) are both zero.

The condition (5.25) implies that DET $\bar{\partial}_{\xi^{\prime}}$ is canonically trivial. The Quillen norm on this space is thus given by

$$
\left\|1_{O(\Theta)}\right\|_{Q}^{2}=\operatorname{det} \bar{\partial}_{\xi^{\prime}}^{\dagger} \bar{\partial}_{\xi^{\prime}}
$$

The condition (5.2) gives that

$$
\operatorname{DET} \bar{\partial}_{\xi} \cong\left(\wedge^{k} H^{0}(\Sigma ; \xi)\right)^{-1}
$$

so $\left(u_{1} \wedge \cdots \wedge u_{k}\right)^{-1}$ is a basis of DET $\bar{\partial}_{\xi}$. Its Quillen norm is

$$
\left\|\left(u_{1} \wedge \cdots \wedge u_{k}\right)^{-1}\right\|_{Q}^{2}=\operatorname{det}^{\prime} \bar{\partial}_{\xi}^{\dagger} \bar{\partial}_{\xi} \cdot\left(\operatorname{det}\left(u_{i}, u_{j}\right)\right)^{-1} .
$$

Finally we see that the formula (5.17) is a consequence of the generalized insertion formula (5.23), of (5.26) and (5.28) and of the following lemma:

\section{Lemma 3.}

$$
I\left(\left(u_{1} \wedge \cdots \wedge u_{k}\right)^{-1}\right)=(2 \pi i)^{-q} \operatorname{Det}\left(u_{i}, P_{j}, Q_{\ell}\right)^{-1}
$$

This lemma is a consequence of the following observations:

i) $H^{1}\left(\Sigma ; \xi \otimes O\left(\sum Q_{i}\right)\right)=0$ because of (5.25). Thus

$$
\operatorname{DET} \bar{\partial}_{\xi \otimes O\left(\sum Q_{i}\right)} \cong\left(\wedge^{p} H^{0}\left(\Sigma ; \xi \otimes O\left(\sum_{i=1}^{q} Q_{i}\right)\right)\right)^{-1}
$$

ii) The map

$$
I_{2}:\left.\left[\operatorname{DET} \bar{\partial}_{\xi \otimes O\left(\sum Q_{i}\right)}\right]^{-1} \longrightarrow \bigotimes_{i=1}^{p} \xi\right|_{P_{i}}
$$

is the $p$-th exterior power of the restriction map

$$
r:\left.\left.H^{0}\left(\Sigma ; \xi \otimes O\left(\sum Q_{i}\right)\right) \rightarrow\left[\xi \otimes O\left(\sum Q_{i}\right)\right]\right|_{\sum P_{j}} \cong \bigoplus_{i=1}^{p} \xi\right|_{P_{i}}
$$


$I_{2}^{\prime}$ is an isomorphism when the insertion points satisfy (5.25), and in that case it is the isomorphism (5.21).

iii) The map $I_{2}:$ DET $\bar{\partial}_{\xi} \stackrel{\sim}{\longrightarrow}$ DET $\bar{\partial}_{\xi \otimes O}\left(\sum Q_{i}\right) \otimes\left[\left.\otimes_{i=1}^{q}\left(\xi^{-1} \otimes K\right)\right|_{Q_{i}}\right]$ can be written, thanks to the identifications (5.27) and (5.29):

$$
\begin{aligned}
I_{1}\left(\left(u_{1} \wedge \cdots \wedge u_{k}\right)^{-1}\right)= & \left(u_{1} \wedge \cdots \wedge u_{k} \wedge 2 \pi i \mathcal{G}\left(\cdot, Q_{1}\right) v_{1} \wedge \cdots \wedge 2 \pi i \mathcal{G}\left(\cdot, Q_{q}\right) v_{q}\right)^{-1} \\
& \otimes\left(v_{1} \otimes \cdots \otimes v_{q}\right)^{-1}
\end{aligned}
$$

for any choice of nonzero $\left.v_{i} \in\left(\xi \otimes K^{-1}\right)\right|_{Q_{i}}$.

The last assertion is a consequence of the fact that $2 \pi i \mathcal{G}\left(\cdot, Q_{j}\right) v_{j}$ is an element of $H^{0}\left(\Sigma ; \xi \otimes O\left(\sum Q_{i}\right)\right)$, which has $\left.\left[\delta_{i j} v_{i}\right]_{i=1, \ldots q} \in \bigoplus_{i=1}^{q}\left(\xi \otimes K^{-1}\right)\right|_{Q_{i}}$ as its image by the residue map at the points $Q_{1}, \ldots, Q_{q}$. See the remark following (4.7).

This completes the proof of (5.17), hence in particular of (4.15) and so the bosonization identity (4.4), when the condition (5.2) is satisfied.

When (5.2) is not satisfied, e.g. for $\xi=K$, the proof of the bosonization formula (5.6) follows the same lines as the proof of (5.17). The only real difference occurs in the construction of the isomorphism $I_{1}$ :

$$
I_{1}: \mathrm{DET} \bar{\partial}_{K} \stackrel{\sim}{\longrightarrow} \mathrm{DET} \bar{\partial}_{K \otimes O}\left(\sum Q_{j}\right)
$$

We need the identifications

$$
\begin{gathered}
\operatorname{DET} \bar{\partial}_{K} \cong\left(\wedge^{g} H^{0}(\Sigma ; K)\right)^{-1} \\
\operatorname{DET} \bar{\partial}_{K \otimes O\left(\sum Q_{j}\right)} \cong\left(\wedge^{g-1+q} H^{0}\left(\Sigma ; K \otimes O\left(\sum Q_{j}\right)\right)\right)^{-1} \\
I_{1}\left(\left(\omega_{1} \wedge \cdots \wedge \omega_{g}\right)^{-1}\right)=(2 \pi i)^{1-q}\left[\omega_{1} \wedge \cdots \wedge \omega_{g} \wedge\left(\mathcal{G}\left(\cdot, Q_{1}\right)-\mathcal{G}\left(\cdot, Q_{q}\right)\right) \wedge \cdots\right. \\
\left.\wedge\left(\mathcal{G}\left(\cdot, Q_{q-1}\right)-\mathcal{G}\left(\cdot, Q_{q}\right)\right)\right]^{-1} .
\end{gathered}
$$

Note that the norm of the constant function 1 appears in the denominator of the LHS of (5.6), not in the numerator as (5.7), (5.8) might seem to imply. This is because we have represented a basis of coker $\bar{\partial}_{K}$ by a basis of the dual space ker $\bar{\partial}$ using Serre duality (2.15).

\section{Acknowledgements}

We would like to thank S. Coleman, S. Dellapietra, V. Dellapietra, D. Freed, J. Harris, D. Kazhdan, E. Martinec, I. Singer, and C. Soulé for many valuable discussions. 


\section{Appendix A: Proof of (2.32)}

We are to establish that Fig. 4 commutes when $F_{A}, H$, and $I_{A}$ are defined as in (2.32), (2.29), and (2.26) respectively. Consider the following complex function on the cut surface $\Sigma_{c}:$

$$
f(P)=\vartheta\left(z+I_{A}\left[P-P_{0}\right] \mid \tau_{A}\right) / \vartheta\left(I_{A}\left[P-P_{0}\right] \mid \tau_{A}\right)
$$

By the Riemann vanishing theorem $f$ has exactly $g$ zeros and poles for generic $z$. The zeros are at $P_{i}$ where

$$
z+I_{A}\left[\sum_{i} P_{i}-D_{A}\right]=0
$$

and similarly the poles, with $z$ replaced by $0 . D_{A}$ is a divisor representing the preferred spin bundle $L_{A}$. Hence

$$
I_{\mathfrak{A}}[\operatorname{div} f]=-z
$$

As $P$ goes around the cycle $n \cdot a+m \cdot b, f$ jumps by $\mathrm{e}^{-2 \pi i m \cdot z}$. Build a bundle $\xi$ with transition functions such that $f$ defines a meromorphic section $\sigma$ on all of $\Sigma$. Next put a norm on $\xi$ : let

$$
\|\sigma\|^{2}=\exp \left\{2 \pi i(z-\bar{z}) \cdot Y_{A} \cdot\left[\int_{P_{0}}^{P} \omega_{A}-\int_{P_{0}}^{P} \bar{\omega}_{A}\right]\right\}|f|^{2},
$$

where $Y_{A}=\left(\tau_{A}-\bar{\tau}_{A}\right)^{-1}$. Note that $\|\sigma\|^{2}$ is the quotient of two of the $\mathcal{N}$ functions defined in (2.43). We then get the connection

$$
\Theta=\partial \log \|\sigma\|^{2}=2 \pi i(z-\bar{z}) Y_{A} \omega_{A}+\partial \log f
$$

Integrating along contours which avoid the poles and zeros of $\sigma$, we find the holonomy

$$
H(\xi)=(\alpha, \beta)\left(\begin{array}{c}
-Y_{A}(z-\bar{z}) \\
-\tau_{A} Y_{A}(z-\bar{z})+z
\end{array}\right)
$$

Applying (2.32) to $H(\xi)$ we recover $-z \in J_{\mathscr{A}}$, which indeed agrees with (5.30). 


\section{References}

[1] S. Coleman, Phys. Rev. D11 (1975) 2088.

[2] R. Marnelius, Nucl. Phys. B211 (1983) 14.

[3] D. Friedan, E. Martinec, and S. Shenker, "Conformal invariance, supersymmetry, and string theory," Nucl. Phys. B271 (1986) 93.

[4] W. Siegel and B. Zwiebach, Nucl. Phys. B263 (1986) 105.

[5] L. Alvarez-Gaumé, J. B. Bost, G. Moore, P. Nelson, and C. Vafa, "Bosonization in arbitrary genus," Phys. Lett. B178 (1986) 41.

[6] L. Alvarez-Gaumé, G. Moore, and C. Vafa, "Theta functions, modular invariance, and strings," Comm. Math. Phys. 106 (1986) 1.

[7] J. Bost and P. Nelson, "Spin-1/2 bosonization on compact surfaces," Phys. Rev. Lett. 57 (1986) 795.

[8] R. Shankar, Phys. Lett. 92B, 333 (1980); E. Witten, in Fourth workshop on grand unification, ed. H. Weldon, P. Langacker, and P. Steinhardt, (Birkhauser, 1983).

[9] R. Nepomechie, "Nonabelian bosonization, triality, and superstring theory," Phys. Lett. 178B (1986) 207; 180B (1986) 423; L. Brown and R. Nepomechie, Non-abelian bosonization: current correlation functions," Washington preprint.

[10] D. Gross, J. Harvey, E. Martinec, and R. Rohm, "The heterotic string," Phys. Rev. Lett. 54 (1985) 502; "Heterotic string theory I, II," Nucl. Phys. B256 (1985) 253; B267 (1986) 75.

[11] D. Friedan, E. Martinec, and S. Shenker, Phys. Lett. B160 (1985) 55.

[12] V. Knizhnik, "Covariant superstring amplitudes from the sum over fermionic surfaces," Phys. Lett. 178B (1986) 21.

[13] D. Quillen, "Determinants of Cauchy-Riemann operators on Riemann surfaces," Funk. Anal. i Prilozen 19, 37 (1985) [=Funct. Anal. Appl. 19, 31 (1986)].

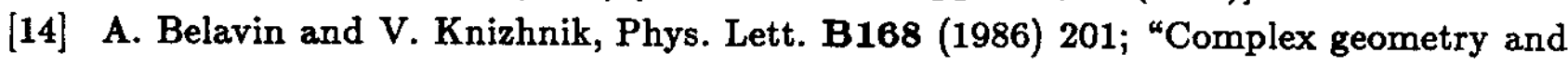
theory of quantum strings," Landau Inst. preprint submitted to ZETF.

[15] J. Bost and J. Jolicœur, Phys. Lett. B174 (1986) 273.

[16] P. Nelson, "Lectures on strings and moduli space" HUTP-86/A047, to appear in Phys. Reports.

[17] J. B. Bost, "Fibrés déterminants régularisés et mesures sur les espaces de modules des courbes complexes," sém. Bourbaki, 1986-7, $\mathrm{n}^{\circ} 676$.

[18] D. Freed, "On determinant line bundles," preprint to appear in Mathematical aspects of string theory, ed. S. T. Yau (World, 1987).

[19] G. Faltings, "Calculus on arithmetic surfaces," Ann. Math. 119 (1984) 387.

[20] S. Arakelov, Izv. Akad. Nauk. SSSR Ser. Mat. 38 (1974) [=Math. USSR Izv. 8 (1974) 1167]. 
[21] E. Martinec, "Conformal field theory on a (super-)Riemann surface," Nucl. Phys. B281 (1987) 157.

[22] T. Eguchi and H. Ooguri, "Chiral bosonization on a Riemann surface," LPTENS 86.39.

[23] E. Verlinde and H. Verlinde, "Chiral bosonization, determinants, and the string partition function," Utrecht preprint.

[24] L. Alvarez-Gaumé, C. Gomez, and C. Reina, "Loop groups, grassmannians, and string theory," CERN Th.4641/87.

[25] I. Frenkel, J. Funct. Anal. 44 (1981) 259.

[26] P. Goddard and D. Olive, in Vertex operators in mathematics and physics, ed. J. Lepowsky, S. Mandelstam, and I. M. Singer (Springer, 1985).

[27] N. Ishibashi, Y. Matsuo, and H. Ooguri, "Soliton equations and free fermions on Riemann surfaces," Tokyo UT-499.

[28] C. Vafa, "Operator formalism on Riemann surfaces," Harvard HUTP-87/A008.

[29] L. Alvarez-Gaumé and P. Nelson, "Riemann surfaces and string theories," CERNTH.4615/86, to appear in Supersymmetry, supergravity, and superstrings '86 (World Scientific, 1986).

[30] V. Knizhnik, "Analytic fields on Riemann surfaces," Phys. Lett. 180B (1986) 247.

[31] A. Redlich, H. Schnitzer, and K. Tsokos, "Bose-fermi equivalence on the twodimensional torus for simply-laced groups," Brandeis BRX TH-203; H. Schnitzer and $\mathrm{K}$. Tsokos, "Partition functions and fermi-bose equivalence for simply-laced groups on compact Riemann surfaces," Brandeis BRX TH-215.

[32] V. Kostelecky, O. Lechtenfeld, W. Lerche, S. Samuel, and S. Watamura, "Conformal techniques, bosonization, and tree-level string amplitudes," CERN-TH.4560/86.

[33] J. Bagger, D. Nemeschansky, N. Seiberg, and S. Yankielowicz, "Bosons, fermions, and Thirring strings," HUTP-86/A088.

[34] M. Dugan and H. Sonoda, "Functional determinants on Riemann surfaces," LBL22776, 1986.

[35] R. Bott, L. Tu Differential forms in algebraic topology, (Springer, 1982).

[36] L. Bers, Bull. Lon. Math. Soc. 4 (1972) 257.

[37] D. Mumford, Tata lectures on theta (Birkhaüser, 1983).

[38] M. Atiyah, "Riemann surfaces and spin structures," Ann. Sci. Ec. Norm. Sup. $4^{e}$ série 4 (1971) 47.

[39] R. O. Wells, Differential analysis on complex manifolds, (Springer, 1980).

[40] O. Alvarez, "Topological quantization and cohomology," Comm. Math. Phys. 100 (1985) 279.

[41] R. Gunning, Lectures on Riemann surfaces (Princeton Univ. Press, 1966).

[42] H. Farkas, I. Kra, Riemann Surfaces (Springer, 1980). 
[43] P. Griffiths and J. Harris, Principles of algebraic geometry, (Wiley, 1978).

[44] J. Igusa, Theta functions (Springer, 1972).

[45] S. Mandelstam, Phys. Rev. D11 (1975) 3026.

[46] D. Wolf and J. Zittartz, Zeit. Phys. B51 (1983) 65.

[47] T. Banks et al., Nucl. Phys. B108 (1976) 119.

[48] C. Vafa, "Modular invariance and discrete torsion on orbifolds," Nucl. Phys. B273 (1986) 592.

[49] H. Sonoda, "Calculation of a propagator on a Riemann Surface," Phys. Lett. 178B (1986) 390.

[50] A. Polyakov, Phys. Lett. $103 B$ (1981) 207.

[51] O. Alvarez, "Theory of strings with boundary," Nucl. Phys. B216 (1983) 125.

[52] J.-B. Bost, talk presented at the $8^{\text {th }}$ International Congress on Mathematical Physics, Marseille, 1986.

[53] J. Fay, Theta functions on Riemann surfaces (Springer, 1973.

[54] J.-M. Bismut and D. Freed. "Geometry of elliptic families I, II," Comm. Math. Phys. 106 (1986) 159; 107 (1986) 103.

[55] F. Knudsen and D. Mumford, "The projectivity of the moduli space of stable curves, I," Math. Scand. 39 (1976) 19.

[56] Séminaire de Géométrie Algèbrique 6, Springer Lecture Notes in Math. 225 (1971).

[57] M. Atiyah and I. Singer, "Dirac operators coupled to vector potentials," Proc. Nat. Acad. Sci. USA, 81 (1984) 2597.

[58] J. Harris, in Proc. Int. Cong. of Mathematicians 1983, Warszawa ed. C. Olech and Z. Ciesielski (Elsevier, 1984). 